\title{
TRIANGULATIONS OF SUBANALYTIC SETS AND LOCALLY SUBANALYTIC MANIFOLDS
}

\author{
BY \\ M. SHIOTA AND M. YOKOI \\ Dedicated to Professor René Thom
}

\begin{abstract}
If two polyhedrons are locally subanalytically homeomorphic (that is, the graph is locally subanalytic), they are PL homeomorphic. A locally subanalytic manifold is one whose coordinate transformations are locally subanalytic. It is proved that a locally subanalytic manifold has a unique PL manifold structure. A semialgebraic manifold also is considered.
\end{abstract}

1. Introduction. One of the main results is Theorem 4.1, which states that a locally subanalytic homeomorphism of polyhedrons is locally subanalytically isotopic to a PL homeomorphism. Consequently, a subanalytic triangulation of a subanalytic set (whose existence is shown in $[\mathbf{3}, \mathbf{5}]$ ) is unique up to a PL homeomorphism. These answer questions posed in $[3$ and 13] in weaker forms. The former result also gives a negative answer to a conjecture in $[\mathbf{1 3}]$ of whether there exists a semialgebraic homeomorphism from $\Pi_{7}^{5}$ to $\mathbf{R} P^{5}$, where $\Pi_{7}^{5}$ is Siebenmann's example of a PL manifold homeomorphic, but not PL homeomorphic, to $\mathbf{R} P^{5}[\mathbf{1 6}]$.

We sketch its proof. Let $h: X \rightarrow Y$ be a locally subanalytic homeomorphism of polyhedrons. We find closed neighborhoods $X_{1}, Y_{1}$ of the singular sets of $h, h^{-1}$ in $X, Y$, respectively, using Whitney stratifications of the singular sets, such that (1) $X_{1}, Y_{1}$ are $C^{\infty}$ triangulable, and (2) $\overline{X-X_{1}}$ and $\overline{Y-Y_{1}}$ are $C^{\infty}$ diffeomorphic and, hence, PL homeomorphic. For (1) we need a generalization of the $C^{\infty}$ triangulation theorem of Cairns-Whitehead. The key lemma to (2) is a version (2.14) of Proposition 5.1 of [14] (about topological equivalence of subanalytic functions). By the Alexander trick we extend the PL homeomorphism $\overline{X-X_{1}} \rightarrow \overline{Y-Y_{1}}$ to the global. We once more use the Alexander trick to find a locally subanalytic isotopy of $h$ to a PL homeomorphism.

We show locally subanalytic triangulations of locally subanalytic sets and Proposition 2.14 in $\S 2$; and we explain our generalization of the Cairns-Whitehead theorem and the Alexander trick in $\S 3$. $\$ 4$ deals with Theorem 4.1 and its proof.

As the set of all locally subanalytic homeomorphisms of open subsets of $\mathbf{R}^{n}$ is a pseudo-group, we can define locally subanalytic manifolds as those whose coordinate transformations are locally subanalytic (§5). We show that every locally subanalytic manifold has a unique PL manifold structure (5.3).

In $\S 6$ we define semialgebraic manifolds. The relation between semialgebraic manifolds and compact PL manifolds possibly with boundary is similar to that between Nash manifolds and compact $C^{\infty}$ manifolds possibly with boundary, as

Received by the editors November 21, 1983.

1980 Mathematics Subject Classification. Primary 32B25; Secondary 32B20.

Key words and phrases. Triangulation, subanalytic, polyhedron, PL homeomorphism.

(C) 1984 American Mathematical Society $0002-9947 / 84 \$ 1.00+\$ .25$ per page 
follows (see [14]). By putting boundary on a semialgebraic manifold, we can compactify it; in other words a noncompact semialgebraic manifold is semialgebraically homeomorphic to the interior of some compact semialgebraic manifold with boundary. As a compact semialgebraic manifold possibly with boundary is semialgebraically homeomorphic to a compact PL manifold possibly with boundary, we have a correspondence from the semialgebraic homeomorphism classes of all semialgebraic manifolds to the PL homeomorphism classes of all compact PL manifolds possibly with boundary. We prove that the correspondence is one-to-one and onto. Accordingly there exist two semialgebraic (=finite) PL manifolds PL homeomorphic but semialgebraically distinct.

As a semialgebraic triangulation of semialgebraic sets is always possible $[\mathbf{5}, \mathbf{6}]$, it is meaningful to study properties of semialgebraic polyhedrons. We see in $\S 7$ that a semialgebraic polyhedron essentially consists of finite cells. A curious property of semialgebraic homeomorphisms is that compact polyhedrons $X_{1}$ and $X_{2}$ are PL homeomorphic if $X_{1} \times \mathbf{R}$ and $X_{2} \times \mathbf{R}$ are semialgebraically homeomorphic (7.5). This is derived from finiteness of semialgebraic maps.

In this paper, manifolds do not have boundary and are separable, unless otherwise specified. A topological manifold with boundary with a system of coordinate neighborhoods $\left\{\left(U_{\alpha}, \psi_{\alpha}\right)\right\}$, such that $\psi_{\alpha}$ are homeomorphic onto open sets of $[0, \infty)^{n}$ and whose coordinate transformations are of class $C^{\infty}$, is called a $C^{\infty}$ manifold with cornered boundary.

\section{Locally subanalytic sets.}

2.1. DEFinition. Let $X$ be a subset of $\mathbf{R}^{n}$ and $U$ an open neighborhood of $X$. We call $X$ subanalytic in $U$ if each point $x \in U$ has an open neighborhood $U^{\prime}$ such that $X \cap U^{\prime}$ is a finite union of sets of the form $\operatorname{Im} f_{1}-\operatorname{Im} f_{2}$, where $f_{1}$ and $f_{2}$ are proper analytic maps from analytic manifolds to $\mathbf{R}^{n}$. If $X$ is subanalytic in some $U$ or in $\mathbf{R}^{n}$, then $X$ is called locally subanalytic or subanalytic, respectively.

We remark that every polyhedron contained in a Euclidean space is locally subanalytic, but a polyhedron might not be subanalytic if it is not closed. (This is the reason why we define the concept "locally subanalytic".)

A continuous map of subanalytic sets is called subanalytic if the graph is subanalytic, and a locally subanalytic map is relatively defined. For example, a PL map of polyhedrons in Euclidean spaces is locally subanalytic. Moreover we define a locally subanalytic map of polyhedrons by PL imbedding the polyhedrons in Euclidean spaces. This definition does not depend on the choice of PL imbeddings because the composition of locally subanalytic maps is locally subanalytic. A homotopy $f_{t}: X \rightarrow Y, t \in[0,1]$, is called (locally) subanalytic if $X \times[0,1] \ni(x, t) \rightarrow f_{t}(x) \in Y$ is (locally) subanalytic.

2.2 Triangulation of locally subanalytic sets. The following is a locally subanalytic version of Propositions 3.1, 3.1' and Remark 3.10 in [15]. As the proof is the same, we omit it.

Let $K$ be a simplicial complex in $\mathbf{R}^{n}$ and $U$ an open neighborhood of the underlying polyhedron $|K|$, where $|K|$ is closed. Let $\left\{X_{i}\right\}$ be a family of subsets of $|K|$ locally finite in $U$ and subanalytic in $U$. Then there exist a subdivision $K^{\prime}$ of $K$ and a locally subanalytic isotopy $\tau_{t}:|K| \rightarrow|K|, t \in[0,1]$, of the identity map such that:

(2.2.1) $\tau_{t}(\sigma)=\sigma$ for any $\sigma \in K$ and $t \in[0,1]$; 
(2.2.2) for any $\sigma \in K^{\prime}$ and $t \in[0,1], \tau_{t}(\operatorname{Int} \sigma)$ is an analytic submanifold of $\mathbf{R}^{n}$ and $\left.\tau_{t}\right|_{\operatorname{Int} \sigma}:$ Int $\sigma \rightarrow \tau_{t}(\operatorname{Int} \sigma)$ is an analytic diffeomorphism, where Int $\sigma$, the interior of $\sigma$, is called an open simplex of $K^{\prime}$; and

(2.2.3) each $X_{i}$ is the image under $\tau_{1}$ of a union of open simplexes of $K^{\prime}$.

Moreover, let $L$ be a subcomplex of $K$. Assume each $X_{i} \cap(|K|-|L|)$ is a union of open simplexes of $K-L$. Then

(2.2.4) for any $t \in[0,1]$ and $\sigma \in K$ with $\sigma \cap|L|=\emptyset$, we have $\sigma \in K^{\prime}$ and $\tau_{t}=$ ident on $\sigma$. (See Remark 3.8 in $[\mathbf{1 5}]$.)

2.3. DEFinition. A stratification of a set $X \subset \mathbf{R}^{n}$ is a partition of $X$ into $C^{\infty}$ submanifolds $X_{i}$ of $\mathbf{R}^{n}$ such that $\left\{X_{i}\right\}$ is locally finite at $X$ and $\bar{X}_{i} \cap X_{j} \neq \emptyset$ implies $\bar{X}_{i} \supset X_{j}$.

A Whitney stratification is a stratification satisfying the Whitney condition (b) (see [8]). An analytic stratification means that all strata are of class $C^{\omega}$. If all strata are subanalytic in an open set $U \subset \mathbf{R}^{n}$, we call the stratification subanalytic in $U$. From now on we omit $U$ in the case $U=\mathbf{R}^{n}$.

Let $X \subset \mathbf{R}^{n}$ be a $C^{\infty}$ submanifold. A tube at $X$ is a triple $T=(|T|, \pi, \rho)$, where $|T|$ is a $C^{\infty}$ tubular neighborhood of $X$ for some Riemannian metric of $\mathbf{R}^{n}, \pi:|T| \rightarrow X$ is the projection, and $\rho$ is a nonnegative $C^{\infty}$ function on $|T|$ such that $\rho^{-1}(0)=X$ and each point $x \in X$ is a nondegenerate critical point of $\left.\rho\right|_{\pi^{-1}(x)}(\rho$ is called a distance function).

It is easily seen (cf. the proof of Lemma 4.11 in [15]) that the above definition agrees with [2].

Let $\left\{X_{i}\right\}$ be a stratification in $\mathbf{R}^{n}$. A controlled tube system for $\left\{X_{i}\right\}$ consists of one tube $T_{i}=\left(\left|T_{i}\right|, \pi_{i}, \rho_{i}\right)$ at each $X_{i}$ such that

(2.3.1) $\pi_{i} \circ \pi_{j}(x)=\pi_{i}(x)$ and $\rho_{i} \circ \pi_{j}(x)=\rho_{i}(x)$ for $x \in\left|T_{i}\right| \cap\left|T_{j}\right| \cap \pi_{j}^{-1}\left(\left|T_{i}\right|\right)$.

Let $\left\{T_{i}\right\}$ be a controlled tube system for a stratification $\left\{X_{i}\right\}$ of $X \subset \mathbf{R}^{n}$. A vector field $\xi$ on $\left\{X_{i}\right\}$ consists of one $C^{\infty}$ vector field $\xi_{i}$ on each $X_{i}$. We call it semicontrolled if, for each $i, j$,

$$
d\left(\left.\rho_{i}\right|_{X_{j}}\right) \xi_{j x}=0 \quad \text { for } x \in X_{j} \cap U_{i}
$$

where $U_{i} \subset\left|T_{i}\right|$ is some neighborhood of $X_{i}$ in $\mathbf{R}^{n}$. If, furthermore,

$$
d\left(\pi_{i} \mid X_{j}\right) \xi_{j x}=\xi_{i \pi_{i}(x)} \text { for } x \in X_{j} \cap U_{i}
$$

$\xi$ is called controlled. If $\xi$ is continuous as a map from $X$ to $\mathbf{R}^{n}$, then we call it continuous.

2.4. Existence of controlled tube system [2, Corollary 2.7, Chapter II]. Every Whitney stratification admits a controlled tube system. Moreover, we have the following (see the proof of the corollary quoted above). Let $\left\{X_{i}\right\}$ be a Whitney stratification in $\mathbf{R}^{n}$ such that $X_{1} \in\left\{X_{i}\right\}$ and $\operatorname{dim} X_{1} \leq \operatorname{dim} X_{i}$ for any $i$, and let $\left\{T_{i}=\left(\left|T_{i}\right|, \pi_{i}, \rho_{i}\right)\right\}$ be a tube system for $\left\{X_{i}\right\}$ not necessarily controlled. Then there exists a controlled tube system $\left\{T_{i}^{\prime}=\left(\left|T_{i}^{\prime}\right|, \pi_{i}^{\prime}, \rho_{i}^{\prime}\right)\right\}$ for $\left\{X_{i}\right\}$ such that for each $i$,

$$
\left|T_{i}^{\prime}\right| \subset\left|T_{i}\right|, \quad \rho_{i}=\rho_{i}^{\prime} \text { on }\left|T_{i}^{\prime}\right| \quad \text { and } \quad \pi_{1}=\pi_{1}^{\prime} \text { on }\left|T_{1}^{\prime}\right| .
$$

Hence we can choose a controlled tube system so that $\rho_{i}$ is the square of the distance function from $X_{i}$ in the usual metric of $\mathbf{R}^{n}$. We call such a tube system canonical.

2.5. Lift of vector fields $\left[\mathbf{1 5}\right.$, Lemma 4.11]. Let $\left\{T_{i}\right\}$ be a controlled tube system for a Whitney stratification $\left\{X_{i}\right\}$ in $\mathbf{R}^{n}$. Assume $X_{1} \in\left\{X_{i}\right\}$. Let $\xi_{1}^{\prime}$ be a $C^{\infty}$ 
vector field on $X_{1}$. Then there exists a continuous controlled vector field $\xi=\left\{\xi_{i}\right\}$ on $\left\{\left(X_{i} \cap U,\left.T_{i}\right|_{X_{i} \cap U}\right)\right\}, U$ an open neighborhood of $X_{1}$, such that $\xi_{1}=\xi_{1}^{\prime}$.

2.6. Flows of vector fields. Let $\left\{X_{i}\right\}$ be a stratification of a locally closed set $X \subset \mathbf{R}^{n},\left\{T_{i}\right\}$ a controlled tube system for $\left\{X_{i}\right\}$, and $\xi=\left\{\xi_{i}\right\}$ a vector field on $\left\{X_{i}\right\}$. For each $i$, let $\theta_{i}: D_{i} \rightarrow X_{i}, D_{i} \subset X_{i} \times \mathbf{R}$, be the maximal $C^{\infty}$ flow defined by $\xi_{i}$. Put $D=\bigcup D_{i}$ and define $\theta: D \rightarrow X$ by $\left.\theta\right|_{D_{i}}=\theta_{i}$ for each $i$. We call $\theta$ the flow of $\xi$.

If $\xi$ is semicontrolled, the flow of $\xi$ clearly has the following property. Let $C$ be a compact subset of one $X_{i}$. Then there is a positive constant $\varepsilon$ such that for any $x \in \pi_{i}^{-1}(C) \cap X$ with $\rho_{i}(x)<\varepsilon, \rho_{i}$ is constant on $\theta(D \cap x \times \mathbf{R}) \cap \pi_{i}^{-1}(C)$.

Corollary 4.7 in $[2$, Chapter II] shows, moreover, that if $\xi$ is controlled then $D$ is open in $X \times \mathbf{R}$ and $\theta$ is continuous.

2.7. Properties of subanalytic sets $[\mathbf{4}, \mathbf{1 5}]$. Let $X, Y \subset \mathbf{R}^{n}$ be subanalytic sets.

(2.7.1) $\bar{X}, X \cap Y$ and $X-Y$ are subanalytic.

(2.7.2) The image of $X$ under a proper subanalytic map from $X$ to a Euclidean space is subanalytic.

(2.7.3) There exists a subanalytic subset $X^{\prime}$ of $X$ with $\operatorname{dim} X^{\prime}<\operatorname{dim} X$ such that $X-X^{\prime}$ is an analytic manifold.

(2.7.4) Assume, moreover, $X$ is a connected analytic manifold. Let $f: \mathbf{R}^{n} \rightarrow \mathbf{R}^{m}$ be an analytic map. Then there exists a subanalytic closed subset $X^{\prime}$ of $X$ such that $\operatorname{dim} X^{\prime}<\operatorname{dim} X$ and the differential $d\left(\left.f\right|_{X}\right)$ has constant rank on $X-X^{\prime}$.

(2.7.5) Assume $X$ and $Y$ are analytic manifolds, $X \cap Y=\emptyset$ and $\bar{X} \supset Y$. Then there exists a closed subanalytic subset $Y^{\prime}$ of $Y$ such that $\operatorname{dim} Y^{\prime}<\operatorname{dim} Y$ and $\left(X, Y-Y^{\prime}\right)$ satisfies the Whitney condition (b).

2.8. LEMMA (SEE $[\mathbf{1 5},(5.3 .6),(5.3 .7)])$. Let $\left\{X_{1}, X_{2}\right\}$ be a subanalytic analytic stratification of a set $X \subset \mathbf{R}^{n}$, and $f_{1}, f_{2}$ subanalytic functions on $X$. Assume $f_{1}^{-1}(0)=f_{2}^{-1}(0)=X_{1}, \bar{X}_{2} \supset X_{1}$ and the restrictions to $X_{2}$ of $f_{1}$ and $f_{2}$ are positive analytic functions. Then the set

$S=\left\{x \in X_{2} \mid a_{1} \operatorname{grad} f_{1 x}+a_{2} \operatorname{grad} f_{2 x}=0\right.$ for some $a_{1}, a_{2} \geq 0$ with $\left.a_{1}+a_{2}>0\right\}$ is empty in a neighborhood of $X_{1}$.

Proof. Considering the graph of $f_{1} \times f_{2}$, we can assume that $f_{1}, f_{2}$ are the restrictions to $X$ of analytic functions $F_{1}, F_{2}$ on $\mathbf{R}^{n}$ respectively. If we prove that $S$ is subanalytic, the lemma follows from (5.3.7) in [15]. Lemma 1.6 of [17] says that the subset of $\mathbf{R}^{n} \times G_{n, m}$ consisting of $\left(x, T X_{2 x}\right), x \in X_{2}$, is subanalytic, where $m=\operatorname{dim} X_{2}, T X_{2}$ is the tangent space, and $G_{n, m}$ is the Grassmann manifold with naturally given affine algebraic structure. It is easy to see that the sets $\left\{\left(H, H^{\prime}\right) \in\right.$ $\left.G_{n, m} \times G_{n, n-m} \mid H \perp H^{\prime}\right\}$ and $\left\{(H, x) \in G_{n, m} \times \mathbf{R}^{n} \mid x \in H\right\}$ are algebraic in $G_{n, m} \times G_{n, n-m}$ and $G_{n, m} \times \mathbf{R}^{n}$ respectively. Hence

$$
\left\{\left(x, T X_{2 x},\left(T X_{2 x}\right)^{\perp}, u, v\right) \mid x \in X_{2}, u \in T X_{2 x}, v \perp T X_{2 x}\right\}
$$

is a subanalytic subset of $\mathbf{R}^{n} \times G_{n, m} \times G_{n, n-m} \times \mathbf{R}^{n} \times \mathbf{R}^{n}$. Take its image $X_{2}^{\prime}$ under the projection $\mathbf{R}^{n} \times G_{n, m} \times G_{n, n-m} \times \mathbf{R}^{n} \times \mathbf{R}^{n} \rightarrow \mathbf{R}^{n} \times \mathbf{R}^{n} \times \mathbf{R}^{n}$. Then

$$
X_{2}^{\prime}=\left\{(x, u, v) \in X_{2} \times \mathbf{R}^{n} \times \mathbf{R}^{n} \mid u \in T X_{2 x}, v \perp T X_{2 x}\right\} .
$$

(2.7.2) says that $X_{2}^{\prime}$ is subanalytic, and so is

$$
X_{2}^{\prime \prime}=\left\{(x, u, v, w) \in X_{2}^{\prime} \times \mathbf{R}^{n} \mid w=u+v\right\} .
$$


Clearly the subset of $\mathbf{R}^{n} \times \mathbf{R}^{n}$ consisting of points $\left(x, \operatorname{grad} F_{1 x}\right)$ is analytic. Hence

$$
\tilde{X}_{2}=\left\{(x, u, v, w) \in X_{2}^{\prime \prime} \mid w=\operatorname{grad} F_{1 x}\right\}
$$

is subanalytic. Now $\left\{\left(x, \operatorname{grad} f_{1 x}\right) \mid x \in X_{2}\right\}$ is the projection image of $\tilde{X}_{2}$ onto the first two factors. Therefore, by (2.7.2), $\left\{\left(x, \operatorname{grad} f_{1 x}\right)\right\}$ is subanalytic and, moreover, so is $\left\{\left(x, \operatorname{grad} f_{1 x}, \operatorname{grad} f_{2 x}\right) \mid x \in X_{2}\right\}$. After considering the subanalytic set

$$
\begin{aligned}
\left\{\left(x, \operatorname{grad} f_{1 x}, \operatorname{grad} f_{2 x}, a_{1}, a_{2}\right) \mid x\right. & \in X_{2}, a_{1} a_{2} \geq 0, \\
& \left.a_{1}+a_{2}=1, a_{1} \operatorname{grad} f_{1 x}+a_{2} \operatorname{grad} f_{2 x}=0\right\},
\end{aligned}
$$

we see in the same way as above that $S$ is subanalytic.

2.9. COROLLARY. Under the same notations and assumptions as 2.8 , there exist a $C^{\infty}$ vector field $v$ on $X_{2}$ and a neighborhood $U$ of $X_{1}$ in $X$ such that $v f_{1}$ and $v f_{2}$ are positive on $U-X_{1}$.

Proof. Define $v$ on $U-X_{1}$ for small $U$ by $\left|\operatorname{grad} f_{2}\right| \operatorname{grad} f_{1}+\left|\operatorname{grad} f_{1}\right| \operatorname{grad} f_{2}$, and extend $v$ to $X_{2}$.

2.10. LEMMA. Let $X \subset \mathbf{R}^{n}$ be a subanalytic set, $\left\{X_{i}\right\}$ its subanalytic analytic Whitney stratification, and $\left\{T_{i}\right\}$ a controlled tube system for $\left\{X_{i}\right\}$. Assume $X_{1} \in$ $\left\{X_{i}\right\}$ and $X_{1} \subset \bar{X}_{i}$ for any $i$. Let $f_{1}, f_{2}$ be the restrictions to $X$ of analytic functions on $\mathbf{R}^{n}$ such that $f_{1}^{-1}(0)=f_{2}^{-1}(0)=X_{1}$ and $f_{1}, f_{2}>0$ on $X-X_{1}$. Then there exist a neighborhood $U$ of $X_{1}$ in $\mathbf{R}^{n}$ and a controlled vector field $\xi=\left\{\xi_{i}\right\}$ on $\left\{X_{i}\right\}_{i \neq 1}$ such that for each $i, \xi_{i} f_{1}$ and $\xi_{i} f_{2}$ are positive on $U \cap X_{i}$.

Proof. Apply 2.9 to $f_{1}, f_{2}$ and $\left\{X_{1}, X_{i}\right\}$ for each $i \neq 1$. Then there exists a vector field $\xi^{\prime}=\left\{\xi_{i}^{\prime}\right\}$ on $\left\{X_{i}\right\}_{i \neq 1}$ such that $\xi_{i}^{\prime} f_{1}$ and $\xi_{i}^{\prime} f_{2}$ are positive on $U \cap X_{i}$ for some neighborhood $U$. Hence the lemma follows from [15, Lemma 4.14]. But we repeat the proof because we later use the same idea of proof.

We assume for simplicity $U=\mathbf{R}^{n}$, the index set $=\{1,2, \ldots\}$ and $\operatorname{dim} X_{i}<$ $\operatorname{dim} X_{i+1}$ for any $i$. We modify $\xi^{\prime}$ so as to be controlled by induction as follows. Let $k \geq 2$. Assume $\left.\xi^{\prime}\right|_{V_{k-1}}$ is controlled for some neighborhood $V_{k-1}$ of $\bigcup_{i=2}^{k-1} X_{i}$. We will modify $\left\{\xi_{i}^{\prime}\right\}_{i \geq k}$ so that $\left.\xi^{\prime}\right|_{V_{k}}$ is controlled for some $V_{k}$. By 2.5 we can lift $\xi_{k}^{\prime}$, namely there exists a continuous controlled vector field $\left\{\xi_{j k}\right\}$ on $\left\{\left(X_{j} \cap\right.\right.$ $\left.\left.W_{k},\left.T_{j}\right|_{X_{j} \cap W_{k}}\right)\right\}_{j \geq k}$ such that $\xi_{k k}=\xi_{k}^{\prime}$ for some neighborhood $W_{k}$ of $X_{k}$. By the continuity of $\left\{\xi_{j k}\right\}$, we can suppose that $\xi_{j k} f_{1}$ and $\xi_{j k} f_{2}$ are positive on $X_{j} \cap W_{k}$. Let $V_{k-1}^{\prime}$ and $W_{k}^{\prime}$ be neighborhoods of $\bigcup_{i=2}^{k-1} X_{i}$ and $X_{k}$, respectively, such that

$$
\bar{V}_{k-1}^{\prime}-(\bar{X}-X)-X_{1} \subset V_{k-1}, \quad \bar{W}_{k}^{\prime}-\left(\bar{X}_{k}-X_{k}\right) \subset W_{k} .
$$

Let $\varphi$ be a $C^{\infty}$ function on $\mathbf{R}^{n}-(\bar{X}-X)-X_{1}$ such that

$$
0 \leq \varphi \leq 1, \quad \varphi^{-1}(1) \supset \overline{\left(W_{k}^{\prime}-V_{k-1}\right)}, \quad \operatorname{supp} \varphi \subset W_{k}-\bar{V}_{k-1}^{\prime},
$$

and for each $x \in X_{k}, \varphi$ is constant on $\pi_{k}^{-1}(x) \cap W_{k}^{\prime}$. This is possible when we choose sufficiently small $V_{k-1}^{\prime}$. Put

$$
\xi_{j}^{\prime \prime}= \begin{cases}\varphi \xi_{j k}+(1-\varphi) \xi_{j}^{\prime} & \text { for } j>k, \\ \xi_{j}^{\prime} & \text { for } j \leq k\end{cases}
$$

Then $\xi_{j}^{\prime \prime} f_{1}$ and $\xi_{j}^{\prime \prime} f_{2}$ are positive on each $X_{j}, j \neq 1$, and it is easy to check also that $\left.\left\{\xi_{j}^{\prime \prime}\right\}\right|_{V_{k}}, V_{k}=V_{k-1}^{\prime} \cup W_{k}^{\prime}$, is controlled. Hence the lemma follows. 
We require the vector field in 2.10 to satisfy the following additional property in exchange for (2.2.3).

2.11. Lemma. Let $X,\left\{X_{i}\right\},\left\{T_{i}\right\}, f_{1}$ and $f_{2}$ be the same as 2.10. Assume $X$ is locally closed in $\mathbf{R}^{n}$ and

$$
X_{1}=\mathbf{R}^{m} \times 0=\left\{\left(x_{1}, \ldots, x_{n}\right) \in \mathbf{R}^{n} \mid x_{m+1}=\cdots=x_{n}=0\right\} .
$$

Then there exist an open neighborhood $U$ of $X_{1}$ and a semicontrolled vector field $\xi=\left\{\xi_{i}\right\}$ on $\left\{X_{i}\right\}_{i \neq 1}$ such that for each $i, \xi_{i} f_{1}$ and $\xi_{i} f_{2}$ are positive on $U \cap X_{i}$ and for each $i$ and $1 \leq l \leq m,\left.\xi_{i}\right|_{\left\{x_{l}=0\right\} \cap X_{i} \cap U}$ is tangent to $\left\{x_{l}=0\right\} \cap X_{i} \cap U$.

To prove this we need the following, which is inspired by the proof of Thom's First Isotopy Lemma (see [2, Theorem 5.2, Chapter II]).

2.12. LEMMA. Let $\left\{X_{i}\right\}$ be a Whitney stratification of a locally closed set $X \subset \mathbf{R}^{n}$ such that $X_{1}=\mathbf{R}^{m} \times 0 \in\left\{X_{i}\right\}, X_{1} \subset \bar{X}_{i}$ for any $i$. Let $\left\{T_{i}\right\}$ be a controlled tube system for $\left\{X_{i}\right\}$. Given $1 \leq m^{\prime} \leq m$. Then we have open neighborhoods $V, W$ and $O$ of 0 in $\mathbf{R}^{m^{\prime}} \times 0\left(\subset \mathbf{R}^{m^{\prime}} \times \mathbf{R}^{n-m^{\prime}}\right), 0 \times \mathbf{R}^{n-m^{\prime}}\left(\subset \mathbf{R}^{m^{\prime}} \times \mathbf{R}^{n-m^{\prime}}\right)$ and $\mathbf{R}^{n}$, respectively, and a homeomorphism $\alpha: V \times W \rightarrow O$ such that: for each $i,\left.\alpha\right|_{V \times X_{i 0}}$ is a diffeomorphism onto $X_{i} \cap O$, where $X_{i 0}=W \cap X_{i}$; for each $i$,

$$
\rho_{i} \circ \alpha(x, y)=\rho_{i}(y), \quad x \in V, y \in W \text { near } X_{i 0}
$$

and for each $1 \leq l \leq m$,

$$
O \cap\left\{x_{l}=0\right\}= \begin{cases}\alpha\left(\left(V \cap\left\{x_{l}=0\right\}\right) \times W\right) & \text { if } l \leq m^{\prime}, \\ \alpha\left(V \times\left(W \cap\left\{x_{l}=0\right\}\right)\right) & \text { if } l>m^{\prime}\end{cases}
$$

ProOF. We consider the case $m^{\prime}=1$. By 2.4 , we have a tube system $\left\{T_{i}^{\prime}=\right.$ $\left.\left(\left|T_{i}^{\prime}\right|, \pi_{i}^{\prime}, \rho_{i}^{\prime}\right)\right\}$ for $\left\{X_{i}\right\}$ such that $\left|T_{i}^{\prime}\right| \subset\left|T_{i}\right|, \rho_{i}=\rho_{i}^{\prime}$ on $\left|T_{i}^{\prime}\right|$ and $\pi_{1}^{\prime}$ is the orthogonal projection. Hence, we may suppose that $\pi_{1}$ is the orthogonal projection. Since $\left\{X_{i}, \mathbf{R}^{n}-\bar{X}\right\}$ is a Whitney stratification and $X$ is locally closed, we also assume $X$ is a neighborhood of $X_{1}$. Apply 2.5 to $\partial /\left.\partial x_{1}\right|_{X_{1}}$. Then we have a controlled vector fied $\xi=\left\{\xi_{i}\right\}$ on $\left\{\left(X_{i} \cap U,\left.T_{i}\right|_{X_{i} \cap U}\right)\right\}$ such that $\xi_{1}=\partial / \partial x_{1}$ for some neighborhood $U$ of $X_{1}$. It follows that

$$
d\left(\rho_{i} \mid X_{j}\right) \xi_{j}=0 \quad \text { on } X_{j} \cap U_{i}
$$

for each $i, j$ and some neighborhood $U_{i}$ of $X_{i} \cap U$, and

$$
d\left(\left.\pi_{1}\right|_{X_{i}}\right) \xi_{i}=\partial / \partial x_{1} \quad \text { on } X_{i} \cap U^{\prime}
$$

for each $i$ and some neighborhood $U^{\prime}$ of $X_{1}$. In particular,

each $\xi_{i}$ is transversal to $\left\{x_{1}=0\right\} \cap U^{\prime}$.

We remark that $\left\{X_{i} \cap\left\{x_{1}=0\right\} \cap U^{\prime \prime}\right\}$ is a Whitney stratification for some neighborhood $U^{\prime \prime}$ of $X_{1}$. Let $\theta$ be the flow of $\xi$, and let $V_{1}$ and $W_{1}$ be open connected small neighborhoods of 0 in $\mathbf{R} \times 0\left(\subset \mathbf{R} \times \mathbf{R}^{n-1}\right)$ and $0 \times \mathbf{R}^{n-1}\left(\subset \mathbf{R} \times \mathbf{R}^{n-1}\right)$, respectively. Define $\alpha_{1}: V_{1} \times W_{1} \rightarrow \mathbf{R}^{n}$ by

$$
\alpha_{1}\left(x_{1}, 0, \ldots, 0, x_{2}, \ldots, x_{n}\right)=\theta\left(0, x_{2}, \ldots, x_{n}, x_{1}\right) .
$$


Put $\alpha_{1}\left(V_{1} \times W_{1}\right)=O_{1}$. Then $\alpha_{1}$ is a homeomorphism onto $O_{1}$ by 2.6 , and $\left.\alpha_{1}\right|_{V_{1} \times X_{i 1}}$ is a diffeomorphism onto $X_{i} \cap O_{1}$ for each $i$ by (2.12.5), where $X_{i 1}=W_{1} \cap X_{i}$. It also follows from (2.12.3) that, for each $i$,

$$
\rho_{i} \circ \alpha_{1}(x, y)=\rho_{i}(y), \quad x \in V_{1}, y \in W_{1} \text { near } X_{i 1},
$$

and, from (2.12.4), that for each $2 \leq l \leq m$,

$$
\alpha_{1}\left(V_{1} \times\left(W_{1} \cap\left\{x_{l}=0\right\}\right)\right)=O_{1} \cap\left\{x_{l}=0\right\} .
$$

It is trivial that $\alpha_{1}\left(0 \times W_{1}\right)=O_{1} \cap\left\{x_{1}=0\right\}$. Hence the lemma for $m^{\prime}=1$ is proved.

If $m^{\prime}=2$ argue in the same way as above about $\left\{X_{i} \cap\left\{x_{1}=0\right\} \cap U^{\prime \prime}\right\}$. Then we have neighborhoods $V_{2}^{\prime}, W_{2}^{\prime}, O_{2}^{\prime}$ of 0 in $0 \times \mathbf{R} \times 0,0 \times 0 \times \mathbf{R}^{n-2}, 0 \times \mathbf{R}^{n-1}$, respectively, and a homeomorphism $\alpha_{2}^{\prime}: V_{2}^{\prime} \times W_{2}^{\prime} \rightarrow O_{2}^{\prime}$ such that the properties corresponding to (2.12.1) and (2.12.2) are satisfied. We choose $V_{2}^{\prime}$ and $W_{2}^{\prime}$ so small that $O_{2}^{\prime} \subset W_{1}$. Put

$$
\begin{gathered}
V_{2}=\left\{\left(x_{1}, x_{2}, 0, \ldots, 0\right) \mid\left(x_{1}, 0, \ldots, 0\right) \in V_{1},\left(0, x_{2}, 0, \ldots, 0\right) \in V_{2}^{\prime}\right\}, \\
W_{2}=W_{2}^{\prime}, \quad O_{2}=\alpha_{1}\left(V_{1} \times O_{2}^{\prime}\right),
\end{gathered}
$$

and define $\alpha_{2}: V_{2} \times W_{2} \rightarrow O_{2}$ by

$$
\alpha_{2}\left(x_{1}, x_{2}, 0, \ldots, 0, x_{3}, \ldots, x_{n}\right)=\alpha_{1}\left(x_{1}, 0, \ldots, 0, \alpha_{2}^{\prime}\left(0, x_{2}, 0, \ldots, 0, x_{3}, \ldots, x_{n}\right)\right) .
$$

Then the lemma for $m^{\prime}=2$ follows. For general $m^{\prime}$, repeating the above argument, we obtain the lemma.

2.13. PROOF OF 2.11. For each subset $I=\left\{i_{1}, \ldots, i_{m^{\prime}}\right\}$ of $\{1, \ldots, m\}$, put

$$
\begin{aligned}
R^{I} & =\left\{\left(x_{1}, \ldots, x_{n}\right) \in \mathbf{R}^{n} \mid x_{i_{1}}=\cdots=x_{i_{m^{\prime}}}=0\right\}, \\
X^{I} & =X \cap R^{I}, \quad\left\{X_{i}^{I}\right\}=\left\{X_{i} \cap R^{I}\right\}, \\
f_{j}^{I} & =\left.f_{j}\right|_{X^{I}} \text { and } \quad \rho_{i}^{I \prime}=\left.\rho_{i}\right|_{\left|T_{i}\right| \cap R^{I}} .
\end{aligned}
$$

Then $\left\{X_{i}^{I}\right\}$ is a subanalytic analytic Whitney stratification of $X^{I}$ in a neighborhood $U^{I}$ of $X_{1}^{I}$ in $R^{I}$, and each $\rho_{i}^{I \prime}$ satisfies the conditions of the distance function (2.3). Hence, by 2.4 , we have a controlled tube system $\left\{T_{i}^{I}=\left(\left|T_{i}^{I}\right|, \pi_{i}^{I}, \rho_{i}^{I}\right)\right\}$ for $\left\{X_{i}^{I} \cap U^{I}\right\}$ such that

$$
\left|T_{i}^{I}\right| \subset\left|T_{i}\right| \cap R^{I} \quad \text { and } \quad \rho_{i}^{I}=\rho_{i} \text { on }\left|T_{i}^{I}\right| .
$$

Apply 2.10 to $\left\{X_{i}^{I} \cap U^{I}\right\},\left\{T_{i}^{I}\right\}, f_{1}^{I}$ and $f_{2}^{I}$, and let $\xi^{I}=\left\{\xi_{i}^{I}\right\}_{i \neq 1}$ be a resultant controlled vector field. If $I=\left\{1, \ldots, m^{\prime}\right\}$, consider the vector field $\left(0, \xi^{I}\right)$ on $\mathbf{R}^{m^{\prime}} \times 0 \times U^{I}\left(\subset \mathbf{R}^{m^{\prime}} \times \mathbf{R}^{n-m^{\prime}} \times \mathbf{R}^{m^{\prime}} \times \mathbf{R}^{n-m^{\prime}}\right)$ and an extension of $\xi^{I} d \alpha\left(0, \xi^{I}\right)$ on $X \cap O^{I}-X_{1}$, where $\alpha$ is given in 2.12 and $O^{I}$ is a neighborhood of 0 in $\mathbf{R}^{n}$. In another case of $I$, by changing coordinates we reduce to the case above. We keep the notation $\xi^{I}$ for the extension. Then we have the following. $\left\{\xi_{i}^{I}\right\}$ is semicontrolled by (2.12.1) and (2.13.1). For each $i$ and $l \in I,\left.\xi_{i}^{I}\right|_{\left\{x_{l}=0\right\} \cap X_{i} \cap O^{I}}$ is tangent to $\left\{x_{l}=0\right\} \cap X_{i} \cap O^{I}$ by (2.12.2). For each $i, \xi_{i}^{I} f_{1}$ and $\xi_{i}^{I} f_{2}$ are positive on $V_{i}^{I} \cap X_{i}$ for some small neighborhood $V_{i}^{I}$ of $X_{i} \cap R^{I} \cap O^{I} / k$ in $\mathbf{R}^{n}$ by 2.10 , where $k$ is a large integer, since $f_{1}$ and $f_{2}$ are restrictions of analytic functions and the extension of $\xi_{i}^{I}$ is of class $C^{\infty}$. Hence, we have to restrict $\xi_{i}^{I}$ to $V_{i}^{I} \cap X_{i}$. Moreover, to avoid

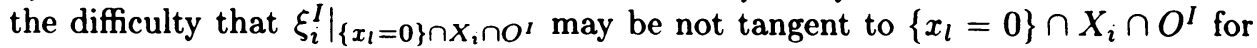


some $l \in I^{c}=\{1, \ldots, m\}-I$, we restrict $\xi_{i}^{I}$ to $V_{i}^{I} \cap X_{i}-\left\{\prod_{i \in I^{c}} x_{i}=0\right\}$. Now we remark that for each $i \neq 1$, at least one of $\xi_{i}^{I}$ is defined at each point of $X_{i}$ near 0 even after the above restriction. Hence, using a $C^{\infty}$ partition of unity in the same way as in the proof of 2.10 , we can contruct a vector field $\xi_{i}$ on each $X_{i}, i \neq 1$, in a neighborhood of 0 so that $\left\{\xi_{i}\right\}$ satisfies the requirement of the lemma. It is easy to define $\xi$ globally once more by a $C^{\infty}$ partition of unity.

2.14. Proposition. Let $\left\{X_{i}\right\}_{i=1, \ldots, m}$ be a subanalytic analytic Whitney stratification of a locally closed set $X \subset \mathbf{R}^{n}$ such that $\operatorname{dim} X_{i}<\operatorname{dim} X_{i+1}$ for any $i$. Let $\left\{T_{i}\right\}$ be a controlled tube system for $\left\{X_{i}\right\}$. Let $f_{1}, f_{2}$ be nonnegative analytic functions on a neighborhood $U$ of $X_{1}$ whose zero sets in $X$ are $X_{1}$. Let $\varphi_{1}, \ldots, \varphi_{k}$ be continuous functions on a neighborhood of $X$, and $Y_{1}, \ldots, Y_{k}$ the respective zero sets. For each $i$, set

$$
X^{+}=X \cap \bigcap_{j}\left\{\varphi_{j} \geq 0\right\}, \quad X_{i}^{+}=X_{i} \cap X^{+} .
$$

Assume that: each $\varphi_{j}$ is analytic and $C^{\infty}$ regular near $Y_{j}$; each $i$ and each subset $\left\{j_{1}, \ldots, j_{k^{\prime}}\right\}$ of $\{1, \ldots, k\}, Y_{j_{1}}$ is transversal to $X_{i} \cap Y_{j_{2}} \cap \cdots \cap Y_{j_{k}}$; and

(2.14.1) $X^{+} \cap\left\{f_{l} \leq \varepsilon\right\}$ is compact for some $\varepsilon>0, l=1$ or 2 .

Then we have a positive number and positive functions $\varepsilon_{1}, \varepsilon_{2}\left(t_{1}\right)$ on $\mathbf{R}, \ldots$, $\varepsilon_{m-1}\left(t_{1}, \ldots, t_{m-2}\right)$ on $\mathbf{R}^{m-2}$ such that for any sequence of positive numbers $\delta_{1} \leq$ $\varepsilon_{1}, \delta_{2} \leq \varepsilon_{2}\left(\delta_{1}\right), \ldots, \delta_{m-1} \leq \varepsilon_{m-1}\left(\delta_{1}, \ldots, \delta_{m-2}\right)$ there exists a $C^{\infty}$ diffeomorphism $\pi$ of $X_{m}$ such that:

(2.14.2) $\{\pi(x) \neq x\}$ is contained in $U \cap U^{\prime}$, where $U^{\prime}$ is a given small neighborhood of $X^{+}$;

$$
\begin{gathered}
\pi\left(X_{m}^{+}-\left\{f_{1} \leq \delta_{1}\right\}-\left\{\rho_{2} \leq \delta_{2}\right\}-\cdots-\left\{\rho_{m-1} \leq \delta_{m-1}\right\}\right) \\
=X_{m}^{+}-\left\{f_{2} \leq \delta_{1}\right\}-\left\{\rho_{2} \leq \delta_{2}\right\}-\cdots-\left\{\rho_{m-1} \leq \delta_{m-1}\right\} \\
\pi\left(X_{m} \cap Y_{j}\right)=X_{m} \cap Y_{j} \text { for any } j .
\end{gathered}
$$

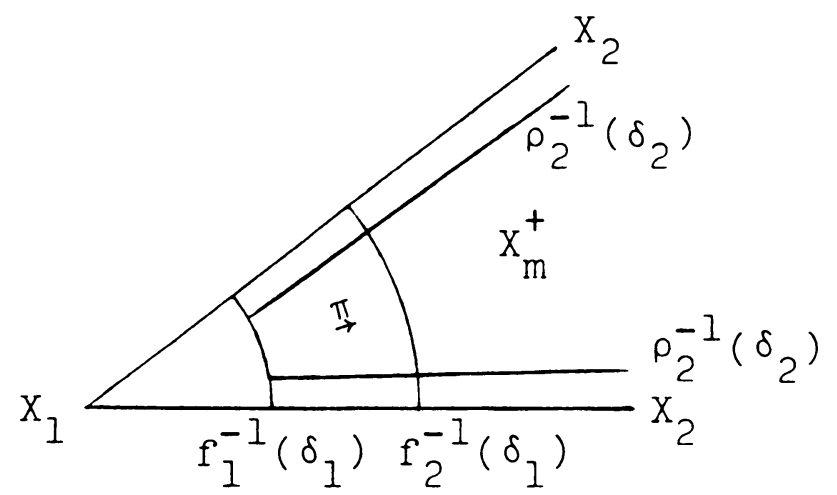

PROOF. By 2.11 there exist open neighborhoods $U_{i}$ of $X_{i}, i=1, \ldots, m-1$, and a $C^{\infty}$ vector field $\xi_{m}$ on $X_{m}$ such that $U_{1} \subset U$,

$$
\xi_{m} f_{1}, \xi_{m} f_{2}>0 \text { on } X_{m} \cap U_{1}
$$




$$
d\left(\left.\rho_{i}\right|_{X_{m}}\right) \xi_{m}=0 \quad \text { on } X_{m} \cap U_{i} \text { for each } i>1
$$

$$
d\left(\left.\varphi_{j}\right|_{X_{m}}\right) \xi_{m}=0 \text { on } X_{m} \cap Y_{j} \text { for each } j .
$$

Let $\theta_{m}: D_{m} \rightarrow X_{m}$ be the flow of $\xi_{m}$. Shrinking $U_{1}$, we can assume by (2.14.1), (2.14.5) and (2.14.7)

$$
\begin{gathered}
X_{m}^{+} \cap U_{1}=X_{m}^{+} \cap\left\{f_{1}<\varepsilon^{\prime}\right\} \quad \text { for some } 0<\varepsilon^{\prime}<\varepsilon, \\
\theta_{m}\left(D_{m} \cap\left(\left(X_{m}^{+} \cap U_{1}\right) \times \mathbf{R}^{-}\right)\right)=X_{m}^{+} \cap U_{1},
\end{gathered}
$$

where $\mathbf{R}^{-}=(-\infty, 0]$, and for each $x_{0} \in X_{m}^{+} \cap \bar{U}_{1}, f_{1} \circ \theta_{m}\left(x_{0}, t\right)$ and $f_{2} \circ \theta_{m}\left(x_{0}, t\right)$ are $C^{\infty}$ regular and increasing on $x_{0} \times\left(t^{-}, 0\right]=D_{m} \cap\left(x_{0} \times \mathbf{R}^{-}\right)$and tend to 0 as $t \rightarrow t^{-}$. Hence, we may regard $X_{m}^{+} \cap U_{1}$ as $\left(0, \varepsilon^{\prime}\right) \times\left(f_{1}^{-1}\left(\varepsilon^{\prime}\right) \cap X_{m}^{+}\right)$and $\left.f_{1}\right|_{X_{m}^{+} \cap U_{1}}$ as the projection onto the first factor.

Choose a small $\varepsilon_{1}>0$ and so that $\varepsilon_{1}<\varepsilon^{\prime}$ and $X_{m}^{+} \cap\left\{f_{2} \leq \varepsilon_{1}\right\} \subset U_{1}$. Let $0 \leq \delta_{1} \leq \varepsilon_{1}$. Then for any point $x \in X_{m}^{+}$with $f_{1}(x)=\delta_{1}$ there exists uniquely $y \in X_{m}^{+}$such that $y \in \theta_{m}\left((x \times \mathbf{R}) \cap D_{m}\right)$ and $f_{2}(y)=\delta_{1}$. Put $\pi(x)=y$. Then $\pi$ is a diffeomorphism from $f_{1}^{-1}\left(\delta_{1}\right) \cap X_{m}^{+}$to $f_{2}^{-1}\left(\delta_{1}\right) \cap X_{m}^{+}$such that

$$
\begin{gathered}
\pi\left(f_{1}^{-1}\left(\delta_{1}\right) \cap X_{m}^{+} \cap Y_{j}\right)=f_{2}^{-1}\left(\delta_{1}\right) \cap X_{m}^{+} \cap Y_{j} \text { for any } j, \\
\rho_{i} \circ \pi=\rho_{i} \quad \text { on } f_{1}^{-1}\left(\delta_{1}\right) \cap X_{m}^{+} \cap U_{i} \text { for any } i
\end{gathered}
$$

by (2.14.6) and (2.14.7); here $U_{i}$ are shrunk if necessary. It is easy to extend $\pi$ by virtue of $\theta_{m}$ to a diffeomorphism of $X_{m}^{+}$so that

$$
\begin{aligned}
& \pi=\text { ident on } X_{m}^{+} \cap\left\{f_{1} \geq \varepsilon^{\prime} \text { or } f_{1} \leq \delta_{1}^{-}\right\}, \\
& \pi\left(X_{m}^{+}-\left\{f_{1} \leq \delta_{1}\right\}\right)=X_{m}^{+}-\left\{f_{2} \leq \delta_{1}\right\}, \\
& \pi\left(X_{m}^{+} \cap Y_{j}\right)=X_{m}^{+} \cap Y_{j} \text { for any } j, \\
& \rho_{i} \circ \pi=\rho_{i} \text { on } X_{m}^{+} \cap U_{i} \text { for any } i>1, \\
& \pi(x) \in \theta_{m}\left(D_{m} \cap(x \times \mathbf{R})\right) \text { for any } x \in X_{m}^{+},
\end{aligned}
$$

where $\delta_{1}^{-}$is an arbitrarily given positive number such that

$$
X_{m}^{+} \cap\left\{f_{1} \leq \delta_{1}^{-}\right\} \subset\left\{f_{2}<\delta_{1}\right\} \text { and } \delta_{1}^{-}<\delta_{1} .
$$

Then (2.14.2) follows from (2.14.8) and, moreover, $\pi$ satisfies (2.14.3). To see this, we remark by (2.14.1) that $X_{2}^{+} \cap\left\{\delta_{1}^{-}-\varepsilon^{\prime \prime} \leq f_{1} \leq \varepsilon^{\prime}+\varepsilon^{\prime \prime}\right\}$ is compact for small $\varepsilon^{\prime \prime}>0$. Hence, we have a small $\varepsilon_{2}>0$ such that

$$
\left\{x \in X_{m}^{+} \mid \delta_{1}^{-} \leq f_{1}(x) \leq \varepsilon^{\prime}, \rho_{2}(x) \leq \varepsilon_{2}\right\} \subset U_{2} .
$$

Let $0<\delta_{2} \leq \varepsilon_{2}$. Then it follows from (2.14.8) and (2.14.9) that $\pi(2.14 .3)^{\prime \prime} \quad \pi\left(X_{m}^{+}-\left\{f_{1} \leq \delta_{1}\right\}-\left\{\rho_{2} \leq \delta_{2}\right\}\right)=X_{m}^{+}-\left\{f_{2} \leq \delta_{1}\right\}-\left\{\rho_{2} \leq \delta_{2}\right\}$.

Repeating this argument for $\rho_{3}, \ldots, \rho_{m-1}$, we obtain equality (2.14.3). By the method of choice of $\delta_{2}, \ldots, \delta_{m-1}$, we clearly have positive functions $\varepsilon_{2}\left(t_{1}\right), \ldots$, $\varepsilon_{m-1}\left(t_{1}, \ldots, t_{m-2}\right)$ required in the proposition.

Moreover, we extend $\pi$ to a diffeomorphism of $X_{m}$ by the use of $\theta_{m}$ so that (2.14.2) remains true. That is easily carried out by $(2.14 .10)$. Then $(2.14 .4)$ holds by $(2.14 .7)$. Hence the proposition is proved. 
2.15. REMARK. In 2.14 the assumption $\operatorname{dim} X_{i}<\operatorname{dim} X_{i+1}$ is intended for clarifying the meaning of 2.14 . If we replace it by the assumption $\operatorname{dim} X_{1}<\operatorname{dim} X_{i}<$ $\operatorname{dim} X_{m}$ for $i \neq 1, m$, then (2.14.3) must be changed by

$$
\pi\left(F_{1}\right)=F_{2}
$$

where

$$
\begin{gathered}
F_{1}=X_{m}^{+}-\left\{f_{1} \leq \delta_{1}\right\}-\left\{\rho_{2} \leq \delta_{l_{2}}\right\}-\cdots-\left\{\rho_{m-1} \leq \delta_{l_{m-1}}\right\} \\
F_{2}=X_{m}^{+}-\left\{f_{2} \leq \delta_{1}\right\}-\left\{\rho_{2} \leq \delta_{l_{2}}\right\}-\cdots-\left\{\rho_{m-1} \leq \delta_{l_{m-1}}\right\}, \\
l_{i}=\operatorname{dim} X_{i}-\operatorname{dim} X_{1}+1 .
\end{gathered}
$$

By the method of construction of $\pi$, the following is clear. For any subset $\left\{i_{1}, \ldots, i_{k}\right\}$ of $\{2, \ldots, m\}$,

$$
\begin{aligned}
\pi\left(F_{1}\right. & \left.\cap\left\{\rho_{i_{1}}=\delta_{l_{i_{1}}}\right\} \cap \cdots \cap\left\{\rho_{i_{k}}=\delta_{l_{i_{k}}}\right\}\right) \\
= & F_{2} \cap\left\{\rho_{i_{1}}=\delta_{l_{i_{1}}}\right\} \cap \cdots \cap\left\{\rho_{i_{k}}=\delta_{l_{i_{k}}}\right\} .
\end{aligned}
$$

3. $C^{\infty}$ triangulations and the Alexander trick. In this section $K$ and $L$ denote simplicial complexes, and $M$ a $C^{\infty}$ submanifold of $\mathbf{R}^{n}$.

3.1. Definition. A $C^{\infty} \operatorname{map} f: K \rightarrow M$ is a map $f:|K| \rightarrow M$ such that $\left.f\right|_{\sigma}, \sigma \in K$, are of class $C^{\infty}$. A linear isomorphism $g: K \rightarrow L$ is a homeomorphism $g:|K| \rightarrow|L|$ carrying each simplex of $K$ linearly onto one of $L$. Let $f: K \rightarrow M$ be a $C^{\infty}$ map and let $b \in|K|$. We define $d f_{b}: \overline{\operatorname{St}}(b, K) \rightarrow \mathbf{R}^{n}$ by

$$
d f_{b}(x)=d\left(\left.f\right|_{\sigma}\right)_{b}(x-b) \quad \text { for } x, b \in \sigma \in K,
$$

where $\overline{\operatorname{St}}(b, K)$ denotes the closed star of $b$ in $K$. We call $f$ a $C^{\infty}$ imbedding if $f$ and $d f_{b}$ are homeomorphisms onto the images for any $b \in|K|$.

A locally finite family $\left\{X_{i}\right\}$ of closed sets in $M$ is called locally $C^{\infty}$ triangulable if each $X_{i}$ is contained in a $C^{\infty}$ submanifold $Y_{i}$ of $M$ of the same dimension so that

(3.1.1) $X_{i}$ is the closure of the union of some connected components of $Y_{i}$ $\bigcup_{\operatorname{dim} Y_{j}<\operatorname{dim} Y_{i}} Y_{j}$,

(3.1.2) each point $a$ of $M$ has a $C^{\infty}$ coordinate system $\varphi: U \rightarrow \mathbf{R}^{m}$ such that $\varphi\left(U \cap Y_{i}\right)$, with $a \in Y_{i}$, are linear subspaces of $\mathbf{R}^{m}$.

For example, $M$ itself, or a $C^{\infty}$ manifold with cornered boundary, is locally $C^{\infty}$ triangulable.

The following shows that a locally $C^{\infty}$ triangulable family is uniquely and globally $C^{\infty}$ triangulable, which is a generalization of the Cairns-Whitehead theorem that a $C^{\infty}$ manifold is uniquely $C^{\infty}$ triangulable. The original proofs also prove the generalization, so we omit the proof (see $[11,10.5,10.6]$ ).

3.2. PROPOSITION. Let $\left\{X_{i}\right\}$ be a locally $C^{\infty}$ triangulable family in $M$. Then there exist $K$ and a $C^{\infty}$ imbedding $f: K \rightarrow M$ such that $f(|K|)=\bigcup_{i} X_{i}$ and each $X_{i}$ is the union of some $f(\sigma)$ 's, $\sigma \in K$ (we call $f$ a $C^{\infty}$ triangulation of $\left\{X_{i}\right\}$ ). Moreover, if $g: L \rightarrow M$ is another $C^{\infty}$ triangulation of $\left\{X_{i}\right\}$, then $K$ and $L$ have subdivisions $K^{\prime}$ and $L^{\prime}$, respectively, and we have a linear isomorphism $h: K^{\prime} \rightarrow L^{\prime}$ such that

$$
f^{-1}\left(X_{i}\right)=(g \circ h)^{-1}\left(X_{i}\right) \text { for any } i .
$$

In 3.2 if $\left\{X_{i}\right\}$ consists of one element and if there is no confusion, we also call $|K|$ a $C^{\infty}$ triangulation of the element. 
3.3. The Alexander trick (e.g. [12]). Let $X, Y$ be compact polyhedrons, $p, q$ be points, and $p * X, q * Y$ be cones. Let $h: p * X \rightarrow q * Y$ be a homeomorphism whose restriction to the base $X$ is PL onto the base $Y$. Then there exists an isotopy $h_{t}, t \in[0,1]$, such that $h_{t} \equiv h$ on $X, t \in[0,1], h_{0} \equiv h$ and $h_{1}$ is a PL homeomorphism. Moreover, if $h$ is subanalytic, $h_{t}$ can be subanalytic, which is easy to see by the definition of subanalytic map.

The following is also clear. Let $G: p * X \rightarrow q * Y$ be a homeomorphism such that $G(X)=Y$. Let $g_{t}: X \rightarrow Y, t \in[0,1]$, be an isotopy of $\left.G\right|_{X}$. Then there exists an isotopy $G_{t}$ of $G$ such that $\left.G_{t}\right|_{X}=g_{t}$. In the case that $G$ and $g_{t}$ are subanalytic, $G_{t}$ can be subanalytic.

4. Piecewise linearization of locally subanalytic homeorphism. In this section we prove the following.

4.1. THEOREM. Let $X, Y$ be polyhedrons, d a metric on $Y, h: X \rightarrow Y$ a locally subanalytic homeomorphism and $\varepsilon$ a positive continuous function on $X$. Then there exists a locally subanalytic isotopy $h_{t}: X \rightarrow Y, t \in[0,1]$, of $h$ such that

(4.1.1) $h_{1}$ is $P L$, and

(4.1.2) $d\left(h(x), h_{t}(x)\right)<\varepsilon(x)$ for any $x \in X$ and $t \in[0,1]$.

Moreover, if $h$ is PL on a neighborhood of a subpolyhedron $X^{\prime}$ of $X$, we can choose the isotopy so that

(4.1.3) $h_{t}(x)=h(x)$ for any $t \in[0,1]$ and any $x$ in a neighborhood of $X^{\prime}$.

Proof. Let $X, Y$ be contained in $\mathbf{R}^{n}$, and $K, L$ be rectilinear triangulations of $X, Y$ respectively. Apply 2.2 to the family of sets $h(\sigma), \sigma \in K$, subanalytic in some open neighborhood of $Y$. Then we can reduce the problem to the case where for each $\sigma \in K, h(\sigma)$ is the underlying polyhedron of some subcomplex of $L$.

Assume for the present that $h(\sigma), \sigma \in K$, are PL balls. Then there exists the required isotopy $h_{t}$ which satisfies, moreover, $h_{t}(\sigma)=h(\sigma)$ for any $t \in[0,1]$ and $\sigma \in K$. We prove this by induction on $m=\operatorname{dim} X$. If $m=0$, it is trivial. Hence we assume it for $\operatorname{dim} \leq m-1$. Let $K^{m-1}$ be the $(m-1)$-skeleton of $K$, and $L^{*(m-1)}$ the subcomplex of $L$ whose underlying polyhedron is $h\left(\left|K^{m-1}\right|\right)$. By the inductive assumption we have a locally subanalytic isotopy $g_{t}:\left|K^{m-1}\right| \rightarrow\left|L^{*(m-1)}\right|$ of $\left.h\right|_{\left|K^{m-1}\right|}$ such that $g_{1}$ is PL and $g_{t}(\sigma)=h(\sigma)$ for any $t$ and $\sigma \in K^{m-1}$, which implies $g_{t}(\partial \sigma)=h(\partial \sigma)$ for any $t$ and $\sigma \in K-K^{m-1}$. Hence, 3.3 shows that $g_{t}$ can be extended to a locally subanalytic isotopy $G_{t}: X \rightarrow Y$ of $h$ and $G_{1}$ is locally subanalytically isotopic to a PL homeomorphism, so the isotopy fixes $\left|K^{m-1}\right|$. Thus we have a locally subanalytic isotopy $h_{t}: X \rightarrow Y$ of $h$ satisfying (4.1.1) and $h_{t}(\sigma)=h(\sigma)$ for any $t \in[0,1]$ and $\sigma \in K$. (4.1.2) follows from (2.2.1) when we choose fine triangulations of $X, Y$, and (4.1.3) follows from (2.2.4). Therefore we only have to prove the weaker Theorem 4.4 below.

4.2. REMARK. In 4.1 if $X, Y$ are contained in $\mathbf{R}^{n}$ as closed sets, then $h_{t}$ is subanalytic.

4.3. COROLLARY. (Hauptvermutung for triangulations of subanalytic or semialgebraic sets). For any locally closed and (locally) subanalytic set $X \subset \mathbf{R}^{n}$, we have a polyhedron in $\mathbf{R}^{n}$, which is (locally resp.) subanalytically homeomorphic to $X$, uniquely up to a $P L$ homeomorphism. The semialgebraic version also holds true (see §6.1). 
4.4. THEOREM. Let $X, Y \subset \mathbf{R}^{n}$ be compact polyhedrons whose local dimensions at any point are constant $m$ and which are subanalytically homeomorphic. Then $X, Y$ are $P L$ homeomorphic.

We prove the theorem in a sequence of lemmas. Let $K, L$ be rectilinear triangulations of $X, Y$, respectively, $h: X \rightarrow Y$ a subanalytic homeomorphism, $Z$ its graph, and $p_{1}: Z \rightarrow X, p_{2}: Z \rightarrow Y$ the projections. Then we have

4.5. LEMMA. There exist a subanalytic analytic Whitney stratification $\left\{Z_{i}\right\}$ of $Z$ and a subanalytic homeomorphim $\chi_{i}: \bar{Z}_{i} \rightarrow B^{m_{i}}$ for each $i$, where $B^{m_{i}}=\{x \in$ $\left.\mathbf{R}^{m_{2}}|| x \mid \leq 1\right\}$ and $m_{i}=\operatorname{dim} Z_{i}$, such that:

(4.5.1) for each $i, j,\left.p_{j}\right|_{z_{2}}$ is a diffeomorphism;

(4.5.2) for each $i, i^{\prime}$ with $Z_{i^{\prime}} \subset \bar{Z}_{i},\left.\chi_{i}\right|_{Z^{\prime}}$ is a diffeomorphism, and $\chi_{i}\left(Z_{i}\right)=$ Int $B^{m_{2}}$;

(4.5.3) for each $i,\left\{\right.$ graph $\left.\chi_{i}\left|Z_{i^{\prime}}\right| Z_{i^{\prime}} \subset \bar{Z}_{i}\right\}$ is a subanalytic analytic Whitney stratification; and

(4.5.4) each $\sigma \in K$ or $\in L$ is the union of some $p_{j}\left(Z_{i}\right)$ 's, $j=1$ or 2 .

PROOF. We remark that (4.5.4) is identical with

(4.5.4)' if $\sigma \in K$ or $\sigma \in L$ intersects with $p_{j}\left(Z_{i}\right)$, then $p_{j}\left(Z_{i}\right) \subset \sigma$.

We inductively prove 4.5 . Let $k \geq 0$. Assume

$(4.5)_{k}$ there is a closed subanalytic subset $Z^{\prime}$ of $Z$ of dimension $\leq k$, a subanalytic analytic Whitney stratification $\left\{Z_{i}\right\}$ of $Z-Z^{\prime}$, and a subanalytic homeomorphism $\chi_{i}: \bar{Z}_{i} \rightarrow B^{m_{2}}$ for each $i$ such that (4.5.1)-(4.5.3) and (4.5.4) hold and $m_{i}>k$ for all $i$.

$(4.5)_{m}$ is trivial, so it is sufficient to show $(4.5)_{k-1}$. If $\operatorname{dim} Z^{\prime}<k,(4.5)_{k-1}$ is identical to $(4.5)_{k}$. Hence we assume $\operatorname{dim} Z^{\prime}=k$. Apply (2.7.1)-(2.7.5) to all $Z^{\prime} \cap \bar{Z}_{i}$ and graph $\left.\chi_{i}\right|_{Z^{\prime} \cap \bar{Z}_{i}}$. Then we have a closed subanalytic subset $Z^{\prime \prime}$ of $Z^{\prime}$ of dimension $<k$ such that: the connected components $Z_{1}^{\prime}, \ldots, Z_{l}^{\prime}$ of $Z^{\prime}-Z^{\prime \prime}$ are subanalytic analytic manifolds; for each $i, j, d\left(p_{j} \mid Z_{i}^{\prime}\right)$ has rank $k$; if $Z_{i^{\prime}}^{\prime} \cap \bar{Z}_{i} \neq \emptyset$ then $Z_{i^{\prime}}^{\prime} \subset \bar{Z}_{i}$; for such a pair $i, i^{\prime},\left.\chi_{i}\right|_{Z^{\prime}}$, is a $C^{\omega}$ diffeomorphism; and for the same $i, i^{\prime},\left(Z_{i}, Z_{i^{\prime}}^{\prime}\right)$ and (graph $\left.\chi_{i}\right|_{Z_{i}}$, graph $\left.\left.\chi_{i}\right|_{Z^{\prime}}\right)$ satisfy the Whitney condition (b). If $p_{j}^{-1}(\operatorname{Int} \sigma) \cap Z_{i}^{\prime}, \sigma \in K$ or $\sigma \in L$, is of dimension $<k$, we add such a set to $Z^{\prime \prime}$. Then (4.5.4) holds for $Z_{1}^{\prime}, \ldots, Z_{l}^{\prime}$. Hence we have shown (4.5) $)_{k-1}$ except for the existence of $\chi_{i}: \bar{Z}_{i}^{\prime} \rightarrow B^{k}$ for each $i$ such that $\left.\chi_{i}\right|_{Z_{i}^{\prime}}$ is a $C^{\omega}$ diffeomorphism onto Int $B^{k}$.

To see that apply 2.2 to each $\left(\bar{Z}_{i}^{\prime}, \bar{Z}_{i}^{\prime} \cap Z^{\prime \prime}=\bar{Z}_{i}^{\prime}-Z_{i}^{\prime}\right)$. Then there exist a simplicial complex $K_{i}$ and a subanalytic homeomorphism $\tau_{i}:\left|K_{i}\right| \rightarrow \bar{Z}_{i}^{\prime}$ such that for earh $\sigma \in K_{i},\left.\tau_{i}\right|_{\text {Int } \sigma}:$ Int $\sigma \rightarrow \tau_{i}($ Int $\sigma)$ is an analytic diffeomorphism and $\bar{Z}_{i}^{\prime} \cap Z^{\prime \prime}$ is the image under $\tau_{i}$ of the underlying polyhedron of a subcomplex of $K_{i}$. Denote by $Z^{\prime \prime}$, the union of the images under $\tau_{i}$ of all simplexes of dimension $k-1$ of $K_{2}$. Thin we have $Z_{i}^{\prime \prime} \supset \bar{Z}_{i}^{\prime} \cap Z^{\prime \prime}$. We remark that there is a subanalytic homeomorphisin! from a $k$-simplex to $B^{k}$ whose restriction to the interior is of class $C^{\omega}$. Honcr, if we replace $Z^{\prime \prime}$ by $\bigcup Z_{i}^{\prime \prime}$, there exist required $\chi_{i}: \bar{Z}_{i}^{\prime} \rightarrow B^{k}$, which proves 4.5 .

Let $I$ be the index set of $\left\{Z_{i}\right\}$. Put

$$
\begin{aligned}
& I^{k}=\left\{i \in I \mid \operatorname{dim} Z_{i} \leq k\right\}, \quad 0 \leq k \leq m-1, \\
& I^{-1}=\emptyset, \quad X_{i}=p_{1}\left(Z_{1}\right) \quad \text { and } \quad Y_{i}=p_{2}\left(Z_{i}\right) \quad \text { for each } i \in I .
\end{aligned}
$$


Then it follows that $\left\{X_{i}\right\}$ and $\left\{Y_{i}\right\}$ are subanalytic analytic Whitney stratifications of $X$ and $Y$, respectively, and $h$ maps $C^{\omega}$ diffeomorphically each $X_{i}$ onto $Y_{i}$. Let $\rho_{i}^{X}, \rho_{i}, \rho_{i}^{Y}$ be the squares of the distance functions from $X_{i}, Z_{i}, Y_{i}$ in $\mathbf{R}^{n}, \mathbf{R}^{n} \times \mathbf{R}^{n}, \mathbf{R}^{n}$, respectively, in the usual metrics, and let $m_{i} \operatorname{denote} \operatorname{dim} X_{i}$. Let $\delta$ be a sequence of positive numbers $\delta_{0}, \ldots, \delta_{m-1}, \gamma: I^{m-1} \rightarrow\{=, \geq\}$ a map, and $\gamma_{0}: I^{m-1} \rightarrow\{\geq\}$ the constant map. Put

$$
\begin{aligned}
& X_{\delta \gamma}=\bigcap_{i \in I^{m-1}}\left\{x \in X \mid \rho_{i}^{X}(x) \gamma(i) \delta_{m_{i}}\right\}, \\
& U_{i \delta}^{X}=\left\{x \in \mathbf{R}^{n} \mid \rho_{i}^{X}(x)<2 \delta_{m_{i}}\right\}-\bigcup_{m_{j}<m_{i}}\left\{\rho_{j}^{X} \leq \delta_{m_{j}} / 2\right\} \quad \text { for } i \in i^{m-1},
\end{aligned}
$$

where $\rho_{i}^{X}(x) \gamma(i) \delta_{m_{i}}$ means $\rho_{i}^{X}(x)=\delta_{m_{i}}$ if $\gamma(i)$ is "=" and $\rho_{i}^{X}(x) \geq \delta_{m_{i}}$ if $\gamma(i)$ is " $\geq$ ". We write $X_{\delta \gamma_{0}}$ briefly as $X_{\delta}$. We similarly define $Z_{\delta \gamma}, U_{i \delta}^{Z}, Z_{\delta}, Y_{\delta \gamma}, U_{i \delta}^{Y}$ and $Y_{\delta}$. Then $X_{\delta \gamma}, Z_{\delta \gamma}$ and $Y_{\delta \gamma}$ are compact subsets of $\bigcup_{i \notin I^{m-1}} X_{i}, \bigcup_{i \notin I^{m-1}} Z_{i}$ and $\bigcup_{i \notin I^{m-1}} Y_{i}$, respectively. Moreover, they are $C^{\infty}$ manifolds with cornered boundary for $\delta$ restricted as follows.

There exist a positive number and positive functions $\varepsilon_{0}, \varepsilon_{1}\left(t_{0}\right)$ on $\mathbf{R}, \ldots$, $\varepsilon_{m-1}\left(t_{0}, \ldots, t_{m-2}\right)$ on $\mathbf{R}^{m-1}$ such that for any sequence $\delta$ of positive numbers, $\delta_{0}, \ldots, \delta_{m-1}$, with $\delta_{0} \leq \varepsilon_{0}, \delta_{1} \leq \varepsilon_{1}\left(\delta_{0}\right), \ldots, \delta_{m-1} \leq \varepsilon_{m-1}\left(\delta_{0}, \ldots, \delta_{m-2}\right)$, and for any $i \in I^{m-1}, \rho_{i}^{X}$ is analytic on $U_{i \delta}^{X}$ and $C^{\infty}$ regular on $U_{i \delta}^{X}-X_{i}$, and for the same $\delta, i$ and any subset $\left\{j_{1}, \ldots, j_{k}\right\}$ of $I^{m-1},\left\{x \in U_{j_{1} \delta}^{X} \mid \rho_{j_{1}}^{X}(x)=\delta_{m_{j_{1}}}\right\}$ is transversal to

$$
X_{i} \cap\left\{x \in U_{j_{2} \delta}^{X} \mid \rho_{j_{2}}^{X}(x)=\delta_{m_{j_{2}}}\right\} \cap \cdots \cap\left\{x \in U_{j_{k} \delta}^{x} \mid \rho_{j_{k}}^{X}(x)=\delta_{m_{j_{k}}}\right\} .
$$

The existence of such $\varepsilon_{0}, \ldots, \varepsilon_{m-1}$ is clear by the definition of analytic Whitney stratification, and similar statements hold for $Z$ and $Y$.

4.6. LEMMA. We can choose $\varepsilon_{0}, \ldots, \varepsilon_{m-1}$ so that for any sequence $\delta$ bounded by $\varepsilon_{0}, \ldots, \varepsilon_{m-1}$ as above, there exists, moreover, a $C^{\infty}$ diffeomorphism $h_{\delta}: X_{\delta} \rightarrow$ $Y_{\delta}$ such that $h_{\delta}\left(X_{\delta \gamma}\right)=Y_{\delta \gamma}$ for any $\gamma: I^{m-1} \rightarrow\{=, \geq\}$.
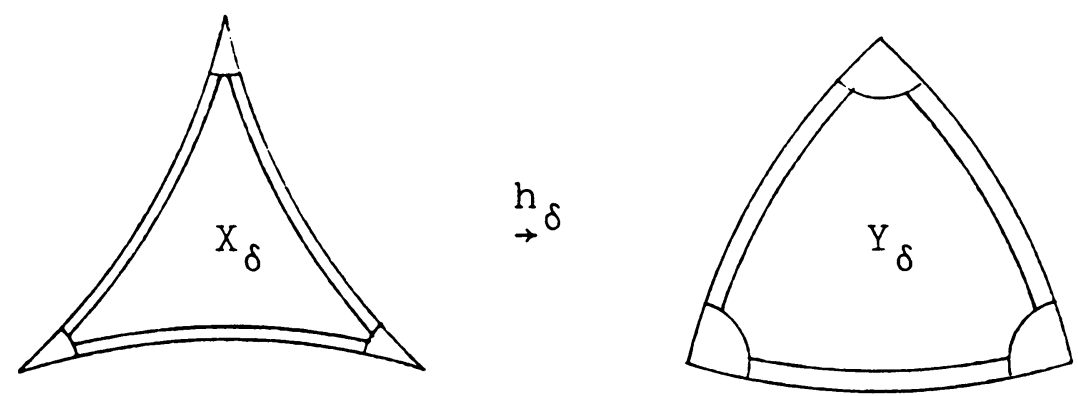

PrOOF. We prove the lemma through the medium of $Z$, namely, we find $C^{\infty}$ diffeomorphisms $p_{1 \delta}: Z_{\delta} \rightarrow X_{\delta}$ and $p_{2 \delta}: Z_{\delta} \rightarrow Y_{\delta}$ such that $p_{1 \delta}\left(Z_{\delta \gamma}\right)=X_{\delta \gamma}$ and $p_{2 \delta}\left(Z_{\delta \gamma}\right)=Y_{\delta \gamma}$ for any $\gamma$. For this we only have to construct a $C^{\infty}$ diffeomorphism $\pi_{\delta}$ of $\bigcup_{i \neq I^{m-1}} Z_{i}$ such that

$$
\pi_{\delta}\left(Z_{\delta \gamma}\right)=p_{1}^{-1}\left(X_{\delta \gamma}\right) \text { for any } \gamma
$$


Put

$$
\psi_{i}=\rho_{i}^{X} \circ p_{1}, \quad Z_{\psi \delta \gamma}=\bigcap_{i \in I^{m-1}}\left\{z \in Z \mid \psi_{i}(z) \gamma(i) \delta_{m_{i}}\right\} .
$$

Then (4.6.1) equals $\pi_{\delta}\left(Z_{\delta \gamma}\right)=Z_{\psi \delta \gamma}$.

We inductively construct $\pi_{\delta}$ as follows. Let $1 \leq l \leq m$. Assume there are $\varepsilon_{0}, \ldots, \varepsilon_{m-1}$ and a $C^{\infty}$ diffeomorphism $\pi_{\delta l}$ of $\bigcup_{i \neq I^{m-1}} Z_{i}$ such that

$$
\pi_{\delta l}\left(\bigcap_{i \in I^{l-1}}\left\{\psi_{i} \gamma(i) \delta_{m_{\jmath}}\right\} \cap \bigcap_{i \in I^{m-1}-I^{l-1}}\left\{\rho_{i} \gamma(i) \delta_{m_{i}}\right\}\right)=Z_{\psi \delta \gamma} .
$$

$(4.6 .1)_{m}$ is trivial, and $(4.6 .1)_{0} \equiv(4.6 .1)$, so it is sufficient to construct $\pi_{\delta(l-1)}$, which satisfies $(4.6 .1)_{l-1}$. For it, after lessening $\varepsilon_{l-1}, \ldots, \varepsilon_{m-1}$, we will construct a $C^{\infty}$ diffeomorphism $\pi_{\delta l}^{\prime}$ of $\bigcup_{m-1} Z_{i}$ such that

$$
\begin{array}{r}
\pi_{\delta l}^{\prime}\left(\bigcap_{i \in I^{l-2}}\left\{\psi_{i} \gamma(i) \delta_{m_{i}}\right\} \cap \bigcap_{i \in I^{m-1}-I^{l-2}}\left\{\rho_{i} \gamma(i) \delta_{m_{i}}\right\}\right) \\
=\bigcap_{i \in I^{l-1}}\left\{\psi_{i} \gamma(i) \delta_{m_{i}}\right\} \cap \bigcap_{i \in I^{m-1}-I^{l-1}}\left\{\rho_{i} \gamma(i) \delta_{m_{i}}\right\}
\end{array}
$$

and

(4.6.3) the closure of $\left\{\pi_{\delta l}^{\prime}(x) \neq x\right\}$ is contained in a small neighborhood of $\bigcup_{i \in I^{l-1}-I^{l-2}} Z_{i}$ because $\pi_{\delta(l-1)}=\pi_{\delta l} \circ \pi_{\delta l}^{\prime}$ satisfies $(4.6 .1)^{l-1}$.

We will choose this neighborhood of $\bigcup_{i \in I^{1-1}-I^{1-2}} Z_{i}$ so small as to be the disjoint union of some small neighborhoods $U_{i}$ of $Z_{i}, i \in I^{l-1}-I^{l-2}$. Hence we can assume $I^{l-1}-I^{l-2}$ consists of one $i$.

Fix positive numbers $\delta_{0}, \ldots, \delta_{l-2}$ so that $\delta_{0} \leq \varepsilon_{0}, \ldots, \delta_{l-2} \leq \varepsilon_{l-2}\left(\delta_{0}, \ldots, \delta_{l-3}\right)$. Consider a subanalytic analytic Whitney stratification

$$
\left\{Z_{j}-V_{l-2}\right\}_{j \in I^{m-1}-I^{l-2}} \cup\left\{\bigcup_{j \notin I^{m-1}} Z_{j}-V_{l-2}\right\},
$$

where

$$
V_{l-2}=\bigcup_{j \in I^{1-2}}\left\{\psi_{j} \leq \delta_{m_{j}} / 2\right\}
$$

a canonical tube system $\left\{T_{j}=\left(\left|T_{j}\right|, \pi_{j},\left.\rho_{j}\right|_{\left|T_{j}\right|}\right)\right\}$ for $\left\{Z_{j}-V_{l-2}\right\}_{j \in I^{m-1}-I^{l-2}}, f_{1}=$ $\rho_{i}, f_{2}=\psi_{i}$ and $\left\{\varphi_{1}, \ldots, \varphi_{k}\right\}=\left\{\psi_{j}-\delta_{m_{j}}\right\}_{j \in I^{l-2}}$. It is easy to check that these satisfy the assumptions in 2.14 or 2.15 . Hence, we have a positive number and positive functions $\varepsilon_{l-1}^{\prime}, \varepsilon_{l}^{\prime}\left(t_{l-1}\right), \ldots, \varepsilon_{m-1}^{\prime}\left(t_{l-1}, \ldots, t_{m-2}\right)$ such that for any sequence of positive numbers

$$
\delta_{l-1} \leq \varepsilon_{l-1}^{\prime}, \delta_{l} \leq \varepsilon_{l}^{\prime}\left(\delta_{l-1}\right), \ldots, \delta_{m-1} \leq \varepsilon_{m-1}^{\prime}\left(\delta_{l-1}, \ldots, \delta_{m-2}\right),
$$

there exists a $C^{\infty}$ diffeomorphism $\pi_{\delta l}^{\prime}$ of $\bigcup_{j \notin I^{m-1}} Z_{j}-V_{l-2}$ such that (4.6.2) is satisfied and

(4.6.4) $\left\{\pi_{\delta l}^{\prime}(x) \neq x\right\}$ is contained in $U_{i}-\bigcup_{j \in I^{1-2}}\left\{\psi_{j} \leq 2 \delta_{m_{j}} / 3\right\}$. Here (4.6.2) follows easily from (2.14.4), (2.15.1) and (2.15.2), and (4.6.4) follows from (2.14.2). Clearly (4.6.4) implies that $\pi_{\delta l}^{\prime}$ is extensible to $\bigcup_{i \notin I^{m-1}} Z_{i}$. As $\varepsilon_{l-1}^{\prime}$ is determined 
by $\delta_{0}, \ldots, \delta_{l-2}$, we regard it as a positive function in $\left(\delta_{0}, \ldots, \delta_{l-2}\right)$-variables, hence $\varepsilon_{l}^{\prime}$ is regarded as a positive function in $\delta_{0}, \ldots, \delta_{l-1}$, and so on. Thus, if we take $\varepsilon_{0}, \ldots, \varepsilon_{l-2}, \min \left\{\varepsilon_{l-1}, \varepsilon_{l-1}^{\prime}\right\}, \ldots, \min \left\{\varepsilon_{m-1}, \varepsilon_{m-1}^{\prime}\right\}$ in place of $\varepsilon_{0}, \ldots, \varepsilon_{m-1}$, then the required $\pi_{\delta l}^{\prime}$ exists.

For each $i, \delta$ and $\gamma$ as above, put

$$
\begin{aligned}
X_{i \delta \gamma} & =\bigcap_{j \in I^{m_{i}-1}}\left\{x \in X_{i} \mid \rho_{j}^{X}(x) \gamma(j) \delta_{m_{j}}\right\}, \\
Y_{i \delta \gamma} & =\bigcap_{j \in I^{m_{i}-1}}\left\{y \in Y_{i} \mid \rho_{j}^{Y}(y) \gamma(j) \delta_{m_{j}}\right\}, \\
X_{i \delta} & =X_{i \delta \gamma_{0}} \text { and } Y_{i \delta}=Y_{i \delta \gamma_{0}} .
\end{aligned}
$$

Then, in the same way as 4.6, we choose $\varepsilon_{0}, \ldots, \varepsilon_{m-1}$ so that for any $i$ and any $\delta$ bounded by $\varepsilon_{0}, \ldots, \varepsilon_{m-1}$, there exists a $C^{\infty}$ diffeomorphism $h_{i \delta}: X_{i \delta} \rightarrow Y_{i \delta}$ such that $h_{i \delta}\left(X_{i \delta \gamma}\right)=Y_{i \delta \gamma}$ for any $\gamma$, and $X_{i \delta \gamma}$ and $Y_{i \delta \gamma}$ are $C^{\infty}$ manifolds with cornered boundary.

Put

$$
\begin{aligned}
& X_{i \delta \gamma}^{+}=\bigcap_{j \in I^{m_{i}-1}}\left\{x \in \mathbf{R}^{n} \mid \rho_{j}^{X}(x) \gamma(j) \delta_{m_{j}}\right\} \cap\left\{x \in \mathbf{R}^{n} \mid \rho_{i}^{X}(x) \leq \delta_{m_{i}}\right\}, \\
& Y_{i \delta \gamma}^{+}=\bigcap_{j \in I^{m_{i}-1}}\left\{y \in \mathbf{R}^{n} \mid \rho_{i}^{Y}(y) \gamma(j) \delta_{m_{j}}\right\} \cap\left\{y \in \mathbf{R}^{n} \mid \rho_{i}^{Y}(y) \leq \delta_{m_{i}}\right\} .
\end{aligned}
$$

Then, for any $\delta$ bounded as above, they are $C^{\infty}$ manifolds with cornered boundary and, moreover, we have the following. Let $\delta$ be fixed, and $x_{\delta}$ (or. $y_{\delta}$ ) be the family of $X_{i \delta \gamma}$ and $X_{i \delta \gamma}^{+}$(or $Y_{i \delta \gamma}$ and $Y_{i \delta \gamma}^{+}$respectively) for all $i \in I$ and for all $\gamma: I^{m-1} \rightarrow\{=, \geq\}$. Then the union of $\chi_{\delta}$ or $Y_{\delta}$ contains $X$ or $Y$, respectively, and $x_{\delta}$ or $y_{\delta}$ is locally $C^{\infty}$ triangulable for the following reason. Let $D$ be the family of all $C^{\infty}$ manifolds $\mathbf{R}^{n}, X_{i} \cap U_{i \delta}^{X}$ and $\left\{x \in U_{i \delta}^{X} \mid \rho_{i}^{X}(x)=\delta_{m_{i}}\right\}, i \in I$, and $D^{\prime}$ the family of all intersections of elements of $D$. Then for any $A_{1}, \ldots, A_{r}$ of $D, A_{1}$ is transversal to $A_{2} \cap \cdots \cap A_{r}$ and, hence, $D^{\prime}$ satisfies (3.1.2). By definition of $X_{i \delta \gamma}$ and $X_{i \delta \gamma}^{+}$, they are defined by $D^{\prime}$ by means (3.3.1). Hence $\chi_{\delta}$ is locally $C^{\infty}$ triangulable.

Furthermore we can easily prove by (4.5.4) that $\mathcal{X}_{\delta} \cup K$ and $Y_{\delta} \cup L$ are locally $C^{\infty}$ triangulable.

4.7. LEMMA. For small $\varepsilon_{0}, \ldots, \varepsilon_{m-1}$, let $i, \delta$ and $\gamma$ be the same as above. $A$ $C^{\infty}$ triangulation of $X_{i \delta \gamma}$ (3.2) is a PL ball.

PROOF. We prove this by induction on $m_{i}$. Let $k \geq 1$. As the case $m_{i}=0$ is trivial, we assume 4.7 for $m_{i}<k$. Let $i_{0} \in I^{k}-I^{k-1}$. Put

$$
J=\left\{i \in I \mid X_{i} \subset \bar{X}_{i_{0}}\right\}, \quad J^{l}=J \cap I^{l} \text { for }-1 \leq l \leq k .
$$

Let $\chi_{i_{0}}: \bar{Z}_{i_{0}} \rightarrow B=B^{k} \subset \mathbf{R}^{k}$ be the homeomorphism in 4.5. Put

$$
B_{i}=\chi_{i_{0}}\left(Z_{i}\right) \quad \text { for } i \in J, \quad B_{\delta \gamma}=\bigcap_{i \in J^{k-1}}\left\{b \in B \mid \rho_{i}^{B}(b) \gamma(i) \delta_{m_{i}}\right\},
$$

where $\rho_{i}^{B}$ are the squares of the distance functions from $B_{i}$ in $\mathbf{R}^{k}$ in the usual metric. Then (4.5.2), (4.5.3) and 4.6 show that for smaill $\varepsilon_{0}, \ldots, \varepsilon_{k-1}, X_{i_{0} \delta \gamma}$ and 
$B_{\delta \gamma}$ are $C^{\infty}$ diffeomorphic. Hence it is sufficient to prove that a $C^{\infty}$ triangulation of $B_{\delta \gamma}$ is a PL ball. In the same way as $\left\{X_{i}\right\}$, we define $B_{i \delta \gamma}, B_{i \delta \gamma}^{+}, B_{\delta}$. We remark that $B_{\delta}$ and $B_{\delta} \cup B$ are locally $C^{\infty}$ triangulable and $\partial B=\bigcup_{i \in J^{k-1}} B_{i}$.

The case $\gamma=\gamma_{0}$ : It is clear that $B \subset \bigcup_{i \in J} B_{i \delta \gamma_{0}}^{+}$, and $B_{i_{0} \delta_{\gamma_{0}}}^{+}=B_{\delta \gamma_{0}}$. For each $i \in J^{k-1}$, we also have

$$
\left(B_{i \delta \gamma_{0}}^{+}, B \cap B_{i \delta \gamma_{0}}^{+}, B \cap B_{i \delta \gamma_{0}}^{+} \cap\left\{\rho_{i}^{B}=\delta_{m_{i}}\right\}, B_{i \delta \gamma_{0}}\right)
$$

is $C^{\infty}$ diffeomorphic to

$$
\left(B^{k-m_{2}}, B^{k-m_{i}} \cap\left\{x_{1} \geq 0\right\}, \partial B^{k-m_{2}} \cap\left\{x_{1} \geq 0\right\}, 0\right) \times B_{i \delta \gamma_{0}},
$$

where

$$
B^{k-m_{i}}=\left\{\left(x_{1}, \ldots, x_{k-m_{i}}\right) \in \mathbf{R}^{k-m_{i}}|| x \mid \leq 1\right\} .
$$

The reason is the following. Let $q: T \rightarrow B_{i \delta \gamma_{0}}$ be the orthogonal projection of a small tubular neighborhood in $\mathbf{R}^{k}$. Recall that for any subset $i_{1}, \ldots, i_{l}$ of $J^{m_{2}-1},\left\{\rho_{i_{1}}^{B}=\delta_{m_{i_{1}}}\right\}$ is transversal to

$$
\left(B_{i}-\bigcup_{m_{j}<m_{i}}\left\{\rho_{j} \leq \delta_{m_{j}} / 2\right\}\right) \cap\left\{\rho_{i_{2}}^{B}=\delta_{m_{j_{2}}}\right\} \cap \cdots \cap\left\{\rho_{i_{l}}=\delta_{m_{i_{l}}}\right\} .
$$

Hence, using $C^{\infty}$ vector fields $\xi$ near $B_{i \delta \gamma_{0}}$ with $d \rho_{i}^{B} \xi=0$ and their $C^{\infty}$ flows, we obtain, in the same way as 2.14 and 4.6 , but more easily, a $C^{\infty}$ diffeomorphism $\tau$ of $\mathbf{R}^{k}$ such that $\left.\tau\right|_{B_{i \delta \gamma_{0}}}=$ ident, $\rho_{i} \circ \tau=\rho_{i}$ near $B_{i \delta \gamma_{0}}$,

$$
\tau\left\{\rho_{j}=\delta_{m}\right\} \cap T=q^{-1}\left(B_{i \delta \gamma_{0}} \cap\left\{\rho_{j}=\delta_{m_{j}}\right\}\right) \quad \text { for any } j \in J^{m_{i}-1},
$$

and for any $x \in B_{i \delta \gamma_{0}}$ and $y \in \tau(\partial B) \cap q^{-1}(x)$, the vector $x y$ is a tangent vector of $\partial B$ at $x$. Now $B_{i \delta \gamma_{0}}$ is homeomorphic to a ball by the induction hypothesis since $B_{i \delta \gamma_{0}}$ and $X_{i \delta \gamma_{0}}$ are $C^{\infty}$ diffeomorphic. Hence the normal bundles of $B_{i \delta \gamma_{0}}$ in $\mathbf{R}^{k}$ and $\partial B$ are trivial, which proves (4.7.1).

By 3.2 we have a $C^{\infty}$ triangulation $g: C \rightarrow \mathbf{R}^{k}$ of $B_{\delta} \cup B$. Let $C_{i}, C_{i}^{\prime}, C_{i}^{\prime \prime}$ be the subcomplexes of $C$ whose underlying polyhedrons are mapped by $g$ onto $B_{i \delta \gamma_{0}}$, $B \cap B_{i \delta \gamma_{0}}^{+}$, and $B \cap B_{i \delta \gamma_{0}}^{+} \cap\left\{\rho_{i}^{B}=\delta_{m_{i}}\right\}$ respectively. Then the induction hypothesis shows that $\left|C_{i}\right|$ are PL balls for $i \in J^{k-1}$. Moreover, (4.7.1) and the uniqueness of $C^{\infty}$ triangulation (3.2) imply that $\left|C_{i}^{\prime}\right|$ and $\left|C_{i}^{\prime \prime}\right|$ are PL balls of dimension $k$ and $k-1$, respertively, for $i \in J^{k-1}$.

We want to see that $\left|C_{i_{0}}^{\prime}\right|=\left|C_{i_{0}}\right|$ is a PL ball. Order $B_{1}, \ldots, B_{i_{0}}$ in such a way that $m_{i} \leq m_{i^{\prime}}$ if $i \leq i^{\prime}$, and regard $B_{\delta \gamma_{0}}$ as obtained by the orderly excisions of $B \cap B_{i \delta \gamma_{0}}^{+}, i=1, \ldots, i_{0}-1$, from $B$. Put

$$
W_{i}=\bigcup_{j \geq i}\left(B \cap B_{j \delta \gamma_{0}}^{+}\right), \quad i=1, \ldots, i_{0} .
$$

Then

$$
\begin{gathered}
W_{i_{0}}=B_{\delta \gamma_{0}}, \quad W_{i}=\left(B \cap B_{i \delta \gamma_{0}}^{+}\right) \cup W_{i+1}, \\
\left(B \cap B_{i \delta \gamma_{0}}^{+}\right) \cap W_{i+1}=B \cap B_{i \delta \gamma_{0}}^{+} \cap\left\{\rho_{i}^{B}=\delta_{m_{i}}\right\}, \quad i=1, \ldots, i_{0}-1 .
\end{gathered}
$$

It is clear that the interior of $B \cap B_{i \delta \gamma_{0}}^{+} \cap\left\{\rho_{i}^{B}=\delta_{m_{i}}\right\}$ is contained in the interior of $W_{i}$ for $i=1, \ldots, i_{0}-1$. Recall the following fact (e.g. $\left.[12,3.13,3.16]\right)$. Let 
$A_{1}, A_{2} \subset \mathbf{R}^{k}$ be polyhedrons such that $A_{1} \cup A_{2}$ and $A_{2}$ are PL $k$-balls and $A_{1} \cap A_{2}$ is a $\mathrm{PL}(k-1)$-ball whose interior is contained in the interior of $A_{1} \cup A_{2}$. Then $A_{1}$ is a PL $k$-ball. From this, it inductively follows that $\bigcup_{j \geq i}\left|C_{j}^{\prime}\right|$ for any $i$ is a PL $k$-ball and, in particular, that $\left|C_{i_{0}}^{\prime}\right|$ is a PL ball. Since a $C^{\infty}$ triangulation is unique, we have shown that any $C^{\infty}$ triangulation of $B_{\delta \gamma_{0}}$ is a PL ball.

The general case of $\gamma$ : We assume $\gamma \neq \gamma_{0}$. At first we remark that if there are $i \neq i^{\prime} \in J$ with $m_{i}=m_{i^{\prime}}$ and with $\gamma(i)=\gamma\left(i^{\prime}\right)==$ then $B_{\delta \gamma}$ is empty. Hence we exclude such a case. Let $i_{1} \in J^{k-1}$ be such that $\gamma\left(i_{1}\right)==$ and $\gamma(i)=\geq$ for any $i \in J^{k-1}-J^{m_{i_{1}}}$. Then we have already proved by the induction hypothesis that a $C^{\infty}$ triangulation of $B \cap B_{i_{1} \delta \gamma}^{+} \cap\left\{\rho_{i_{1}}^{B}=\delta_{m_{i_{1}}}\right\}$ is a PL ball. As

$$
B \cap B_{i_{1} \delta \gamma}^{+} \cap\left\{\rho_{i_{1}}^{B}=\delta_{m_{i_{1}}}\right\}=\bigcap_{j \in I^{m_{i_{1}-1}}}\left\{b \in B \mid \rho_{j}^{B} \gamma(j) \delta_{m_{j}}\right\} \cap\left\{\rho_{i_{1}}^{B}=\delta_{m_{i_{1}}}\right\},
$$

we obtain $B_{\delta} \gamma$ by removing $B \cap B_{i \delta \gamma}^{+}, i \in J^{k-1}-J^{m_{i_{1}}}$, in order from $B \cap B_{i_{1} \delta \gamma}^{+} \cap$ $\left\{\rho_{i_{1}}^{B}=\delta_{m_{i_{1}}}\right\}$ in exactly the same way as in the case $\gamma=\gamma_{0}$. Hence we see that a $C^{\infty}$ triangulation of $B_{\delta \gamma}$ is a PL ball. We complete the proof of 4.7.

4.8. REMARK. For each $i \in I^{m-1}, \delta$ and $\gamma$ as above, a $C^{\infty}$ triangulation of $X_{i \delta \gamma}^{+}$ is a PL ball, and that of $X \cap X_{i \delta \gamma}^{+}$is PL homeomorphic to a PL cone whose base corresponds to the union of $X \cap X_{i \delta \gamma}^{+} \cap\left\{\rho_{i}^{X}=\delta_{m_{i}}\right\}$ and all $X \cap X_{i \delta \gamma^{\prime}}^{+}$such that $X_{i \delta \gamma^{\prime}} \subset \partial X_{i \delta \gamma}$. We also have

$$
X \cap X_{i \delta \gamma}^{+} \cap\left\{\rho_{i}^{X}=\delta_{m_{i}}\right\}=X \cap X_{i \delta \gamma}^{+} \cap \bigcup_{m_{i}<m_{j}} X_{j \delta \gamma_{0}}^{+} .
$$

PROOF. Let $q: T \rightarrow X_{i \delta \gamma_{0}}$ be the orthogonal projection of a small tubular neighborhood in $\mathbf{R}^{n}$, and $x_{0}$ a point of $X_{i \delta \gamma_{0}}$. Then we have seen in the proof of 4.7 that $q$ is trivial and that there is a $C^{\infty}$ diffeomorphism $\tau$ of $\mathbf{R}^{n}$ such that

$$
\begin{aligned}
& \left.\tau\right|_{X_{i \delta \gamma_{0}}}=\text { ident, } \quad \rho_{i} \circ \tau=\rho_{i} \quad \text { near } X_{i \delta \gamma_{0}}, \\
& \tau\left\{\rho_{j}^{X}=\delta_{m_{j}}\right\} \cap T=q^{-1}\left(X_{i \delta \gamma_{0}} \cap\left\{\rho_{j}^{X}=\delta_{m_{j}}\right\}\right) \quad \text { for } j \in I^{m_{i}-1} .
\end{aligned}
$$

Now (4.5.4) means that $X_{i}$ is contained in one open simplex of $K$, say $\operatorname{Int} \sigma_{0}$. Hence, by the method of construction of $\tau$, we can assume $\tau(\sigma)=\sigma$ for $\sigma \in K$, which implies $\tau(X)=X$. Therefore we have a $C^{\infty}$ diffeomorphism $\tau^{\prime}: X_{i \delta \gamma_{0}}^{+} \rightarrow$ $q^{-1}\left(x_{0}\right) \times X_{i \delta \gamma_{0}}$ such that for any $\gamma$

$$
\begin{aligned}
& \left.\tau^{\prime}\right|_{X_{i \delta \gamma}}=x_{0} \times \text { ident, } \\
& \tau^{\prime}\left(\operatorname{Int} \sigma_{0} \cap X_{i \delta \gamma}^{+}\right)=\left(\operatorname{Int} \sigma_{0} \cap q_{i}^{-1}\left(x_{0}\right)\right) \times X_{i \delta \gamma}, \\
& \tau^{\prime}\left(X_{i \delta \gamma}^{+}\right)=q^{-1}\left(x_{0}\right) \times X_{i \delta \gamma}, \\
& \quad \tau^{\prime}\left(X \cap X_{i \delta \gamma}^{+}\right)=\left(X \cap q^{-1}\left(x_{0}\right)\right) \times X_{i \delta \gamma} .
\end{aligned}
$$

Here (4.8.2) easily follows from (4.8.1) and the homogeneity property of $X$ at Int $\sigma_{0}$. Hence a $C^{\infty}$ triangulation of $X_{i \delta \gamma}^{+}$is a PL ball, and that of $X \cap X_{i \delta \gamma}^{+}$is PL homeomorphic to a PL cone since a product of $\mathrm{PL}$ cones is a PL cone. The other statements in the remark are clear.

4.9. Proof OF 4.4. Let $\delta$ be fixed so that the above statements hold. As $\chi_{\delta} \cup K$ and $y_{\delta} \cup L$ are locally $C^{\infty}$ triangulable, by 3.2 we have simplicial complexes $K^{\prime}, L^{\prime}$ 
and $C^{\infty}$ imbeddings $f: K^{\prime} \rightarrow \mathbf{R}^{n}, g: L^{\prime} \rightarrow \mathbf{R}^{n}$ such that $f\left(\left|K^{\prime}\right|\right)=X, g\left(\left|L^{\prime}\right|\right)=Y$, and each of $X_{i \delta \gamma}, X \cap X_{i \delta \gamma}^{+}, \sigma \in K, Y_{i \delta \gamma}, Y \cap Y_{i \delta \gamma}^{+}$and $\sigma \in L$ is a union of some $f\left(\sigma^{\prime}\right), \sigma^{\prime} \in K^{\prime}$, or $g\left(\sigma^{\prime}\right), \sigma^{\prime} \in L^{\prime}$. In particular, we can regard $f$ and $g$ as $C^{\infty}$ triangulations of $K$ and $L$ respectively. Hence, by the uniqueness of $C^{\infty}$ triangulation (3.2), $\left|K^{\prime}\right|$ and $\left|L^{\prime}\right|$ are PL homeomorphic to $X$ and $Y$ respectively. Therefore we need only prove that $\left|K^{\prime}\right|$ and $\left|L^{\prime}\right|$ are PL homeomorphic. Let $K_{i \delta \gamma}, K_{i \delta \gamma}^{+}$be the subcomplexes of $K^{\prime}$ whose underlying polyhedrons are mapped by $f$ to $X_{i \delta \gamma}, X \cap X_{i \delta \gamma}^{+}$, respectively, and let $L_{i \delta \gamma}, L_{i \delta \gamma}^{+}$be similarly defined. Then 4.7 says that $\left|K_{i \delta \gamma}\right|$ and $\left|L_{i \delta \gamma}\right|$ are PL balls. Order the indexes in $I, 1,2, \ldots$ so that $m_{1} \geq m_{2} \geq \cdots$.

We inductively construct a PL homeomorphism from $\left|K^{\prime}\right|$ to $\left|L^{\prime}\right|$ as follows. Let $i \geq 1$. Assume a PL homeomorphism

$$
h_{i}: \bigcup_{j \leq i}\left|K_{j \delta \gamma_{0}}^{+}\right| \rightarrow \bigcup_{j \leq i}\left|L_{j \delta \gamma_{0}}^{+}\right|
$$

such that for any $j \leq i$ and any $\gamma$,

$$
h_{i}\left(\left|K_{j \delta \gamma}^{+}\right|\right)=\left|L_{j \delta \gamma}^{+}\right| \text {. }
$$

If $m_{i}=m$, the existence of $h_{i}$ follows from 4.6 and from the uniqueness of the $C^{\infty}$ triangulation. Hence, we assume $m_{i+1}<m$, and it is sufficient to extend $h_{i}$ to

$$
h_{i+1}: \bigcup_{j \leq i+1}\left|K_{j \delta \gamma_{0}}^{+}\right| \rightarrow \bigcup_{j \leq i+1}\left|K_{j \delta \gamma_{0}}^{+}\right|
$$

so that $h_{i+1}$ satisfies (4.9.1) for any $j \leq i+1$ and any $\gamma$. For any $\gamma, 4.8$ shows that $\left|K_{(i+1) \delta \gamma}^{+}\right|$is PL homeomorphic to a PL cone so that the union of $\left|K_{(i+1) \delta \gamma}^{+}\right| \cap$ $\bigcup_{j \leq i}\left|K_{j \delta \gamma_{0}}^{+}\right|$and all $\left|K_{(i+1) \delta \gamma^{\prime}}^{+}\right|$such that $\left|K_{(i+1) \delta \gamma^{\prime}}\right| \subset \partial\left|K_{(i+1) \delta \gamma}\right|$ corresponds to the base of the cone. Hence we regard $\left|K_{(i+1) \delta \gamma}^{+}\right|$as a PL cone with base the above union. We remark that

$$
\left|K_{(i+1) \delta \gamma}^{+}\right| \cap \bigcup_{j \leq i}\left|K_{j \delta \gamma_{0}}^{+}\right|=\bigcup_{j \leq i}\left|K_{j \delta \gamma_{i+1}}^{+}\right|
$$

where

$$
\gamma_{i+1}(j)= \begin{cases}\gamma(j) & \text { for } j>i+1 \\ = & \text { for } j=i+1 \\ \geq & \text { for } j<i+1\end{cases}
$$

Clearly these hold true for $L_{j \delta \gamma}$ and $L_{j \delta \gamma}^{+}$, and we also regard $\left|L_{(i+1) \delta \gamma}^{+}\right|$as PL cones. Order the elements of $\left\{\gamma: I^{m-1} \rightarrow\{=, \geq\} \mid K_{(i+1) \delta \gamma} \neq \emptyset\right\}, \gamma^{1}, \gamma^{2}, \ldots$, so that $\operatorname{dim} K_{(i+1) \delta \gamma^{1}} \leq \operatorname{dim} K_{(i+1) \delta \gamma^{2}} \leq \cdots$.

We also use induction for the extension of $h_{i}$. Let $0 \leq l$, and assume a PL extension of $h_{i}$,

$$
h_{i l}: \bigcup_{k \leq l}\left|K_{(i+1) \delta \gamma^{k}}^{+}\right| \cup \bigcup_{j \leq i}\left|K_{j \delta \gamma_{0}}^{+}\right| \rightarrow \bigcup_{k \leq l}\left|L_{(i+1) \delta \gamma^{k}}^{+}\right| \cup \bigcup_{j \leq i}\left|L_{j \delta \gamma_{0}}^{+}\right|
$$

such that

$$
h_{i l}\left(\left|K_{(i+1) \delta \gamma^{k}}^{+}\right|\right)=\left|L_{(i+1) \delta \gamma^{k}}^{+}\right| \text {for } k \leq l \text {. }
$$


Now it follows from the cone structures of $\left|K_{(i+1) \delta \gamma^{l+1}}^{+}\right|$and $\left|L_{(i+1) \delta \gamma^{l+1}}^{+}\right|$that the bases of the cones are contained in the domain or the target of $h_{i l}$. Hence 3.3 shows a PL extension of $h_{i l}$ :

$$
h_{i(l+1)}: \bigcup_{k \leq l+1}\left|K_{(i+1) \delta \gamma^{k}}^{+}\right| \cup \bigcup_{j \leq i}\left|K_{j \delta \gamma_{0}}^{+}\right| \rightarrow \bigcup_{k \leq l+1}\left|L_{(i+1) \delta \gamma^{k}}^{+}\right| \cup \bigcup_{j \leq i}\left|L_{j \delta \gamma_{0}}^{+}\right| .
$$

It is trivial that

$$
h_{i(l+1)}\left(\left|K_{(i+1) \delta \gamma^{l+1}}^{+}\right|\right)=\left|L_{(i+1) \delta \gamma^{l+1}}^{+}\right| .
$$

Thus by induction we obtain a required PL extension $h_{i+1}$ of $h_{i}$, which completes the proof of 4.4 .

4.10. REMARK. In 4.1 , given a locally finite point set $\left\{a_{1}, \ldots\right\}$ in $X$, we can clearly choose $h_{t}, t \in[0,1]$, so that $h_{t}\left(a_{i}\right)=h\left(a_{i}\right)$ for any $i$.

5. Locally subanalytic manifolds. It is clear by definition that the set of all locally subanalytic homeomorphisms of open subsets of a Euclidean space is a pseudo-group. Denote it by $\Gamma$.

5.1. DEFINITION. A manifold with $\Gamma$-structure is called a locally subanalytic manifold. A continuous map $f: M_{1} \rightarrow M_{2}$ of locally subanalytic manifolds is called locally subanalytic if for each pair $h_{1}: U_{1} \rightarrow \mathbf{R}^{n}$ and $h_{2}: U_{2} \rightarrow \mathbf{R}^{m}$ of coordinate systems of $M_{1}$ and $M_{2}, h_{2} \circ f \circ h_{1}^{-1}: h_{1}\left(U_{1} \cap f^{-1}\left(U_{2}\right)\right) \rightarrow \mathbf{R}^{m}$ is locally subanalytic.

5.2. REMARK. (i) A PL manifold is a locally subanalytic manifold. (ii) The composition of two subanalytic homeomorphisms is not necessarily subanalytic. Hence the set of subanalytic homeomorphisms of open subsets of $\mathbf{R}^{n}$ is not a pseudogroup. (iii) A topological manifold contained in $\mathbf{R}^{n}$ as a subanalytic subset is not necessarily a locally subanalytic manifold, e.g., Edwards' example of a polyhedron [1] which is homeomorphic to $\mathbf{R}^{n}$ but has no PL manifold structure is not a locally subanalytic manifold by 4.1 and 5.3 .

5.3. THEOREM. For any locally subanalytic manifold $M, M$ is locally subanalytically homeomorphic to a PL manifold. If there are two locally subanalytic homeomorphisms $f_{1}: M \rightarrow N_{1}$ and $f_{2}: M \rightarrow N_{2}$ onto $P L$ manifolds, $f_{2} \circ f_{1}^{-1}: N_{1} \rightarrow N_{2}$ is locally subanalytically isotopic to a PL homeomorphism.

For the proof we need

5.4. LEMMA. $M$ is locally subanalytically homeomorphic to some closed subanalytic subset of a Euclidean space.

Proof. The case of compact $M$ : Clearly we have a finite open covering $\left\{U_{i}\right\}$ of $M$ and a locally subanalytic homeomorphism $h_{i}: U_{i} \rightarrow \mathbf{R}^{n}$ for each $i$. We can assume that $\left\{h_{i}^{-1}(O)\right\}$ is a covering of $M$ where $O=\left\{x \in \mathbf{R}^{n}|| x \mid<1\right\}$. Let $\varphi: \mathbf{R}^{n} \rightarrow \mathbf{R}^{n+1}$ be a subanalytic map such that $\left.\varphi\right|_{O}: O \rightarrow \varphi(O)$ is a homeomorphism and $\varphi\left(\mathbf{R}^{n}-O\right)$ is a point. Let $H_{i}: M \rightarrow \mathbf{R}^{n+1}$ be the extension of $\varphi \circ h_{i}$ defined by $H_{i}\left(M-U_{i}\right)=\varphi\left(\mathbf{R}^{n}-O\right)$. Then $\prod_{i} H_{i}(M)$ is a subanalytic subset of a Euclidean space, and $\prod_{i} H_{i}$ is a subanalytical homeomorphism.

The noncompact case: The case is shown in the same way as 2.10 in $[11]$ (a $C^{\infty}$ imbedding of a $C^{\infty}$ manifold in a Euclidean space).

ProOF OF 5.3. By 2.2 and 5.4, $M$ is locally subanalytically homeomorphic to a polyhedron $N$. We need only prove that $N$ is a PL manifold. For any point $x$ 
of $N$, by Definition 5.1 there is an open neighborhood $U$ of $x$ locally subanalytically homeomorphic to $\mathbf{R}^{n}$. As $U$ itself is a polyhedron, 4.1 shows that $U$ is $\mathrm{PL}$ homeomorphic to $\mathbf{R}^{n}$. Hence $U$ and, consequently, $N$ are PL manifolds.

6. Semialgebraic manifolds. See $[7]$ for the fundamental properties of semialgebraic sets. Let $\operatorname{St}(x, K)$ denote the open star of $x$ in a simplicial complex $K$.

6.1. DEFINITION. A subset of $\mathbf{R}^{n}$ is called semialgebraic if it is a finite union of sets of the form

$$
\left.\left\{x \in \mathbf{R}^{n} \mid f_{1} x\right)>0, \ldots, f_{k}(x)>0, f_{k+1}(x)=0, \ldots, f_{l}(x)=0\right\},
$$

where $f_{i}$ are polynomials on $\mathbf{R}^{n}$. A continuous map of semialgebraic sets is called semialgebraic if the graph is semialgebraic.

For example, a compact polyhedron in a Euclidean space is a semialgebraic set, and a PL map of compact $\mathrm{F}$. 'rons in Euclidean spaces is semialgebraic. We can define a semialgebraic map of compact polyhedrons without particular imbedding of the polyhedrons in Euclidean spaces (see 2.1).

Let $\Gamma$ denote the pseudo-group of all semialgebraic homeomorphisms of semialgebraic open subsets of a Euclidean space. A manifold with $\Gamma$-structure is called a semialgebraic manifold if there is a finite system of coordinate neighborhoods. A semialgebraic map of semialgebraic manifolds is defined in the same way as a locally subanalytic map (5.1).

A compact PL manifold or the interior of a compact PL manifold with boundary is a semialgebraic manifold. Conversely, we have

6.2. THEOREM. Let $M$ be a semialgebraic manifold. Then there exists a compact PL manifold possibly with boundary in some Euclidean space whose interior is semialgebraically homeomorphic to $M$.

ProOF. Assume $M$ is not compact, because the compact case follows more easily. Let $h_{i}: U_{i} \rightarrow \mathbf{R}^{n}, U_{i} \subset M, i=1, \ldots, k$, be a system of coordinate neighborhoods of $M$. For each $i$ put

$$
\begin{aligned}
\varphi_{i}(x) & =1 / \operatorname{dist}\left(x, \overline{h_{i}\left(U_{i}\right)}-h_{i}\left(U_{i}\right)\right), \quad x \in h_{i}\left(U_{i}\right), \\
h_{i}^{\prime} & =\left(h_{i}, \varphi_{i} \circ h_{i}\right) .
\end{aligned}
$$

Then $\varphi_{i}$ and, hence, $h_{i}^{\prime}$ are semialgebraic, $h_{i}^{\prime}$ is a homeomorphism from $U_{i}$ onto the image, and the image is closed in $\mathbf{R}^{n+1}$. Recall that $\mathbf{R}^{n+1}$ is algebraically homeomorphic to $S^{n+1}$ - a point by the stereographic projection. Hence we have a semialgebraic map $h_{i}^{\prime \prime}: U_{i} \rightarrow \mathbf{R}^{n+2}$ such that $h_{i}^{\prime \prime}: U_{i} \rightarrow h_{i}^{\prime \prime}\left(U_{i}\right)$ is a homeomorphism, $h_{i}^{\prime \prime}\left(U_{i}\right)$ is bounded in $\mathbf{R}^{n+2}$, and for every point sequence $\left\{x_{j}\right\}$ of $U_{i}$ such that any subsequence does not converge in $U_{i},\left\{h_{i}^{\prime \prime}\left(x_{j}\right)\right\}$ converges to a point $a_{i} \notin h_{i}^{\prime \prime}\left(U_{i}\right)$. By the last condition we can extend $h_{i}^{\prime \prime}$ to $M$ by putting $h_{i}^{\prime \prime}(x)=a_{i}$ for $x \notin U_{i}$. Let $\tilde{h}_{i}^{\prime \prime}$ be the extension, and put $h=\prod_{i=1}^{k} \tilde{h}_{i}^{\prime \prime}: M \rightarrow \mathbf{R}^{k(n+2)}$. Then $h$ is semialgebraic and a homeomorphism onto the image, $h(M)$ is bounded and semialgebraic, and $\overline{h(M)}-h(M)$ is a point. Hence we can reduce the problem to the case where $M$ is contained in $\mathbf{R}^{m}$ as a bounded semialgebraic subset and where $\bar{M}-M$ is a point $a$.

By Theorem 3 of $[6]$ we have a compact polyhedron $X \subset \mathbf{R}^{m}$ and a semialgebraic homeomorphism $f: \bar{M} \rightarrow X$. The proof of 5.3 says that $X-f(a)$ is a PL manifold. 
Let $K$ be a rectilinear triangulation of $X$ such that $f(a) \in K$, and $K^{\prime}$ is the barycentric subdivision of $K$. Then $X-\operatorname{St}\left(f(a), K^{\prime}\right)$ is a compact PL manifold with boundary whose interior is semialgebraically homeomorphic to $M$.

In 6.2 we denote by $C(M)$ the PL manifold possibly with boundary.

6.3. THEOREM. Let $M_{1}, M_{2}$ be semialgebraic manifolds. The following are equivalent.

(i) $M_{1}, M_{2}$ are semialgebraically homeomorphic.

(ii) $C\left(M_{1}\right), C\left(M_{2}\right)$ are semialgebraically homeomorphic.

(iii) $C\left(M_{1}\right), C\left(M_{2}\right)$ are $P L$ homeomorphic.

ProOF. (iii) $\Rightarrow\left(\right.$ ii) $\Rightarrow$ (i) are trivial. Hence we prove (i) $\Rightarrow$ (iii). We assume $M_{1}$ and $M_{2}$ are not compact, because the compact case is trivial by 4.1 . At first we imbed $C\left(M_{1}\right), C\left(M_{2}\right)$ in $\mathbf{R}^{m}$ for some $m$ so that there are cones $N_{1}, N_{2}$ with vertexes $a_{1}, a_{2} \in \mathbf{R}^{m}$ and bases $\partial C\left(M_{1}\right), \partial C\left(M_{2}\right)$, respectively, such that $C\left(M_{1}\right) \cap N_{1}=$ $\partial C\left(M_{1}\right)$ and $C\left(M_{2}\right) \cap N_{2}=\partial C\left(M_{2}\right)$. Then we have PL maps $\psi_{i}: C\left(M_{1}\right) \rightarrow$ $C\left(M_{1}\right) \cup N_{1}$ and $\psi_{2}: C\left(M_{2}\right) \rightarrow C\left(M_{2}\right) \cup N_{2}$ such that $\psi_{1} \mid C\left(M_{1}\right)-\partial C\left(M_{1}\right)$ and $\left.\psi_{2}\right|_{C\left(M_{2}\right)-\partial C\left(M_{2}\right)}$ are homeomorphisms onto $C\left(M_{1}\right) \cup N_{1}-a_{1}$ and $C\left(M_{2}\right) \cup N_{2}-a_{2}$, respectively, and $\psi_{1}\left(\partial C\left(M_{1}\right)\right)=a_{1}$ and $\psi_{2}\left(\partial C\left(M_{2}\right)\right)=a_{2}$. As $\psi_{1}$ and $\psi_{2}$ are semialgebraic, so are $\left.\psi_{1}\right|_{C\left(M_{1}\right)-\partial C\left(M_{1}\right)}$ and $\left.\psi_{2}\right|_{C\left(M_{2}\right)-\partial C\left(M_{2}\right)}$. Hence (i) implies a semialgebraic homeomorphism $h: C\left(M_{1}\right) \cup N_{1}-a_{1} \rightarrow C\left(M_{2}\right) \cup N_{2}-a_{2}$.

Clearly the extension $\tilde{h}: C\left(M_{1}\right) \cup N_{1} \rightarrow C\left(M_{2}\right) \cup N_{2}$ of $h$ defined by $\tilde{h}\left(a_{1}\right)=a_{2}$ is a homeomorphism, and its graph is the closure of the graph of $h$. Now the closure of a semialgebraic set is semialgebraic [7]. Accordingly $\tilde{h}$ is semialgebraic. Apply 4.1 and 4.10 to $\tilde{h}$. Then we have rectilinear triangulations $K_{1}, K_{2}$ of $C\left(M_{1}\right) \cup$ $N_{1}, C\left(M_{2}\right) \cup N_{2}$, respectively, and a linear isomorphism (3.1) $g: K_{1} \rightarrow K_{2}$ such that $a_{1} \in K_{1}, a_{2} \in K_{2}$ and $g\left(a_{1}\right)=a_{2}$. Hence there is a PL homeomorphism from $C\left(M_{1}\right) \cup N_{1}-\operatorname{St}\left(a_{1}, K_{1}\right)$ to $C\left(M_{2}\right) \cup N_{2}-\operatorname{St}\left(a_{2}, K_{2}\right)$ because of $g\left(\operatorname{St}\left(a_{1}, K_{1}\right)\right)=$ $\operatorname{St}\left(a_{2}, K_{2}\right)$. It is clear that $C\left(M_{1}\right)$ and $C\left(M_{2}\right)$ are PL homeomorphic to $C\left(M_{1}\right) \cup$ $N_{1}-\operatorname{St}\left(a_{1}, K_{1}\right)$ and $C\left(M_{2}\right) \cup N_{2}-\operatorname{St}\left(a_{2}, K_{2}\right)$ respectively. Therefore $C\left(M_{1}\right)$ and $C\left(M_{2}\right)$ are PL homeomorphic.

6.4. COROLLARY. Let $S$ be the semialgebraic homeomorphism classes of all semialgebraic manifolds, $P$ the $P L$ homeornorphism classes of all compact $P L$ manifolds possibly with boundary, and $C: S \rightarrow \mathcal{P}$ the map induced by $C()$. Then $C$ is one-to-one and onto.

PROOF. Trivial by 6.2 and 6.3 .

6.5. REMARK. Let $P^{\prime}$ be the PL homeomorphism classes of interiors of compact PL manifolds possibly with boundary, and $i: P \rightarrow \mathcal{P}^{\prime}$ the natural map. Then $i$ is not one-to-one $[\mathbf{1 0}]$. In other words there are two compact PL manifolds with boundary in a Euclidean space whose interiors are PL homeomorphic but semialgebraically homeomorphic.

6.6. THEOREM. Let $M, N$ be semialgebraic manifolds, $f: M \rightarrow N$ a semialgebraic map, and $h: N \rightarrow C(N)-\partial C(N)$ a semialgebraic homeomorphism (6.2). Then we have a semialgebraic homeomorphism $g: M \rightarrow C(M)-\partial C(M)$ such that $h \circ f \circ g^{-1}$ is extensible to $C(M) \rightarrow C(N)$ as a semialgebraic map.

ProOF. Imbedding $C(N)$ in a Euclidean space, we may assume that $N=\mathbf{R}^{k}$ and $f(M)$ is bounded, and it is sufficient to prove that $f \circ g^{-1}$ is extensible to $C(M)$. 
Moreover, by 6.2 we can assume that $M$ is contained in $\mathbf{R}^{m}$ as a semialgebraic bounded subset. Let $M_{1}$ be the graph of $f$, and $p_{1}, p_{2}$ be the projections of $M_{1}$ to $\mathbf{R}^{m}, \mathbf{R}^{k}$ respectively. Then by $[6]$ we have a compact polyhedron $X \subset \mathbf{R}^{m} \times \mathbf{R}^{k}$, a subpolyhedron $Y$ and a semialgebraic homeomorphism $h:(X, Y) \rightarrow\left(\bar{M}_{1}, \bar{M}_{1}\right.$ $\left.M_{1}\right)$. In the proof of 6.2 we have already shown that $X-Y$ is a PL manifold. Let $(K, L)$ be a rectilinear triangulation of $(X, Y)$ such that $L$ is a full subcomplex of $K$ (see $[12]$ for the definition). Define a simpiicial map $\eta: K \rightarrow\{[0,1], 0,1\}$ by putting $\eta(v)=0$ for vertexes $v \in L$ and $\eta(v)=1$ for other vertexes. Then $[\mathbf{1 2}$, $3.10]$ and the proof of $[\mathbf{1 2}, 3.8]$ tell us that $\left|\eta^{-1}([\varepsilon, 1])\right|$ for any $0<\varepsilon<1$ is a compact PL manifold with boundary $\left|\eta^{-1}(\varepsilon)\right|$. It is easy to see also that there is a PL map $\chi:\left|\eta^{-1}([\varepsilon, 1])\right| \rightarrow X$ such that $\left.\chi\right|_{\left|\eta^{-1}((\varepsilon, 1])\right|}$ is a homeomorphism onto $X-Y$. Then $\left.p_{1} \circ h \circ \chi\right|_{\left|\eta^{-1}((\varepsilon, 1])\right|}$ is a semialgebraic homeomorphism onto $M$, and $\left.f \circ p_{1} \circ h \circ \chi\right|_{\left|\eta^{-1}((\varepsilon, 1])\right|}$ is extensible to $\left|\eta^{-1}([\varepsilon, 1])\right|$ because of $f \circ p_{1} \circ h \circ \chi=p_{2} \circ h \circ \chi$ on $\left|\eta^{-1}((\varepsilon, 1])\right|$. Hence, $g=\left(p_{1} \circ h \circ \chi\right)^{-1}$ is a required homeomorphism.

\section{Semialgebraic polyhedrons and semialgebraic homeomorphisms.}

7.1. Definition. A set of the form

$$
\left\{x \in \mathbf{R}^{n} \mid f_{1}(x) \geq 0, \ldots, f_{k}(x) \geq 0, f_{k+1}(x)=0, \ldots, f_{l}(x)=0\right\},
$$

where $f_{i}$ are linear functions, is called a cell (we do not assume compactness as usual). We define a cell complex in the same way as the case of compact cells.

7.2. Lemma. Let $X \subset \mathbf{R}^{n}$ be a finite union of cells. Then the closure of a connected component of $\mathbf{R}^{n}-X$ is also a finite union of cells.

ProOF. There is a finite cell complex $K$ such that $|K|=\mathbf{R}^{n}$ and $X$ is a union of cells of $K$ for the following reason. Let $X$ be the union of cells $X_{1}, \ldots, X_{m}$, and let each $X_{i}$ be described by linear functions $f_{i 1}, \ldots, f_{i l}$. Let $\alpha=\left(\alpha_{1}, \alpha_{2}, \alpha_{3}\right)$ be a partition of $\{1, \ldots, m\} \times\{1, \ldots, l\}$. Put

$$
\begin{aligned}
X_{\alpha}= & \cap\left\{f_{i j} \leq 0 \text { for }(i, j) \in \alpha_{1}\right\} \cap\left\{f_{i j}=0 \text { for }(i, j) \in \alpha_{2}\right\} \\
& \cap\left\{f_{i j} \geq 0 \text { for }(i, j) \in \alpha_{3}\right\} .
\end{aligned}
$$

Then the collection $K$ of all $X_{\alpha}$ satisfies the required properties (see $[11,7.3,7.5]$ ). Since a cell is closed and connected, and since $\mathbf{R}^{n}$ is the disjoint union of all open cells of $K$, a connected component of $\mathbf{R}^{n}-X$ is a union of open cells of $K$. Hence, the lemma follows.

We prove in the same way

7.3. LEMMA. Let $X_{1}, \ldots, X_{m}$ be cells in $\mathbf{R}^{n}$. Then we have a finite cell complex $K$ such that $|K|=\bigcup_{i=1}^{m} X_{i}$ and each $X_{i}$ is a union of cells of $K$.

7.4. THEOREM. Let $X, Y \subset \mathbf{R}^{n}$ be semialgebraic polyhedrons and $f: X \rightarrow Y$ a semialgebraic $P L$ map. If $X$ is closed in $\mathbf{R}^{n}$, it is a finite union of cells $X_{i}$. In the nonclosed case, $X$ is a finite union of connected components $X_{i}$ of sets of the form $C-(\bar{X}-X), C$ being cells. We can choose such decompositions $\left\{X_{i}\right\}$ and $\left\{Y_{i}\right\}$ of $X$ and $Y$, respectively, so that for each $i, f\left(X_{i}\right) \subset Y_{j}$ and $\left.f\right|_{X_{i}}$ is linear for some $j$.

Proof. We treat only the case where $X$ and $Y$ are closed in $\mathbf{R}^{n}$, because the nonclosed case needs more complicated notation. Let the dimension of $X$ be $r$. We 
prove the first statement of the theorem by induction on $r$. If $r=0$, it is trivial, so we assume the statement for dimension $<r$. Let $K$ be a rectilinear triangulation of $X$, and $X^{\prime}$ the singular set of $X$, namely, consisting of points where the germ of $X$ is of dimension $<r$ or not $C^{\infty}$ smooth. Clearly $X^{\prime}$ is closed, and we know it is semialgebraic [7]. Let $\sigma \in K$, and assume a point of Int $\sigma$ is contained in $X^{\prime}$. Then, by the homogeneity of $X$ at Int $\sigma$, any point of Int $\sigma$ is contained in $X^{\prime}$, which means $X^{\prime}$ is a subpolyhedron of $X$. Hence, by the induction hypothesis $X^{\prime}$ is a finite union of cells.

Now we will see that the closure of each connected component of $X-X^{\prime}$ is a finite union of cells. Let $W$ be such a component. Since $W$ is at once a connected $C^{\infty}$ manifold of dimension $r$ and a union of open simplexes of $K$, we have a plane $P$ of dimension $r$ containing $W$. Hence, $W$ is a connected component of $P-X^{\prime}$. Obviously, $P \cap X^{\prime}$ is a semialgebraic polyhedron of dimension $<r$. Therefore, by the induction hypothesis and 7.2, the closure of $W$ is a finite union of cells. Now the number of connected components of a semialgebraic set is finite. Hence $X$ is a finite union of cells.

For the last statement, consider the graph $Z$ of $f$. As $Z$ is a closed semialgebraic polyhedron in $\mathbf{R}^{n} \times \mathbf{R}^{n}$, the first statement implies a cell decomposition $\left\{Z_{i}^{\prime}\right\}$ of $Z$. Let $p_{1}$ or $p_{2}$ be the projection of $\mathbf{R}^{n} \times \mathbf{R}^{n}$ to the first or second factor, respectively, and put $Y_{i}^{\prime}=p_{2}\left(Z_{i}^{\prime}\right)$ for each $i$. Now we can prove in the same way as the compact cell case that $Y_{i}^{\prime}$ are cells. By 7.3 we have a cell decomposition $\left\{Y_{i}\right\}$ of $Y$ such that each $Y_{i}^{\prime}$ is a union of some $Y_{i}^{\prime}$ 's. Put $\left\{Z_{i}\right\}=\left\{Z_{j}^{\prime} \cap P_{2}^{-1}\left(Y_{k}\right)\right\}$ and $X_{i}=p_{1}\left(Z_{i}\right)$ for each $i$. Then $\left\{X_{i}\right\}$ is a cell decomposition of $X$, each $f\left(X_{i}\right)$ is contained in some one $Y_{j}$, and $\left.f\right|_{X_{i}}$ is linear. Hence the theorem is proved.

7.5. THEOREM. Let $X_{1}, X_{2} \subset \mathbf{R}^{n}$ be compact polyhedrons. If $X_{1} \times \mathbf{R}, X_{2} \times$ $\mathbf{R} \subset \mathbf{R}^{n} \times \mathbf{R}$ are semialgebraically homeomorphic, $X_{1}$ and $X_{2}$ are $P L$ homeomorphic.

PROOF. Since $\mathbf{R}$ is semialgebraically homeomorphic to $(0,1) \subset \mathbf{R}, X_{1} \times(0,1)$ and $X_{2} \times(0,1) \subset \mathbf{R}^{n} \times \mathbf{R}$ are semialgebraically homeomorphic. Let $Y_{1}, Y_{2}$ be open cones in $\mathbf{R}^{n} \times \mathbf{R}$ with vertex $0=0 \times 0$ and bases $X_{1} \times 1, X_{2} \times 1$ respectively. Then we have natural semialgebraic homeomorphisms from $X_{1} \times(0,1), X_{2} \times(0,1)$ to $Y_{1}-0, Y_{2}-0$ respectively. Hence there is a semialgebraic homeomorphism $f: Y_{1}-0 \rightarrow Y_{2}-0$. Here we can assume without loss of generality that $f(x) \rightarrow 0$ as $x \rightarrow 0$. Let $\tilde{f}$ be the extension of $f$ to $Y_{1}$ defined by $\tilde{f}(0)=0$. Then we see in the same way as the proof of 6.2 that $\tilde{f}$ is semialgebraic. Hence, by 4.1 and $4.10,\left(Y_{1}, 0\right)$ and $\left(Y_{2}, 0\right)$ are PL homeomorphic. Now the links of 0 in rectilinear triangulations of $Y_{1}$ and $Y_{2}$ are PL homeomorphic to $X_{1}$ and $X_{2}$ respectively. Therefore the theorem follows.

7.6. EXAMPLE. In 7.5, if $X_{1}$ and $X_{2}$ are not compact, they are not necessarily PL homeomorphic as follows. Let $Y$ be Mazur's example [9] of a compact contractible PL manifold with boundary of dimension 4 whose boundary is not simply connected, and let $X_{1}$ be the interior of $Y$. Assume $Y$ is PL imbedded in $\mathbf{R}^{n}$. Let $X_{2}$ be an open 4-simplex in $\mathbf{R}^{n}$. Then it is known that $Y \times[0,1]$ and $\bar{X}_{2} \times[0,1]$ are PL and, hence, semialgebraically homeomorphic. Hence $X_{1} \times(0,1), X_{2} \times(0,1) \subset \mathbf{R}^{n} \times \mathbf{R}$ are semialgebraically homeomorphic. But $X_{1}$ and $X_{2}$ are not homeomorphic, because $X_{1}$ is not simply connected at infinity. 


\section{REFERENCES}

1. R. D. Edwards, The double suspension of a certain homology 3-sphere is $S^{5}$ (unpublished).

2. C. G. Gibson et al, Topological stability of smooth mappings, Lecture Notes in Math., vol. 552, Springer-Verlag, Berlin and New York, 1976.

3. R. M. Hardt, Triangulation of subanalytic sets and proper light subanalytic maps, Invent. Math. 38 (1977), 207-217.

4. H. Hironaka, Subanalytic sets, number theory, algebraic geometry and commutative algebra, in honour of $Y$. Akizuki, Kinokuniya, Tokyo, 1973, pp. 453-493.

5. - Triangulations of algebraic sets, Proc. Sympos. Pure Math., vol. 29, Amer. Math. Soc., Providence, R.I., 1975, pp. 165-185.

6. S. Lojasiewicz, Triangulations of semi-analytic sets, Ann. Scuola Norm. Sup. Pisa Cl. Sci. 18 (1964), 449-474.

7. , Ensembles semi-analytique, Inst. Hautes Etudes Sci., Paris, 1965.

8. J. N. Mather, Stratifications and mappings, Dynamical Systems (M. M. Peixoto, ed.). Academic Press, New York, 1973, pp. 195-223.

9. B. Mazur, A note on some contractible 4-manifolds, Ann. of Math. (2) 73 (1961), 221-228.

10. J. Milnor, Two complexes which are homeomorphic but combinatorially distinct, Ann. of Math. (2) 74 (1961), 575-590.

11 J. R. Munkres, Elementary differential topology, Ann. of Math. Studies, no. 61, Princeton Univ. Press, Princeton, N.J., 1968.

12. C. P. Rourke and B. J. Sanderson, Introduction to pieceurise-linear topology, Ergebnisse Math. Grenzgebiete, Bd. 69, Springer-Verlag, Berlin and New York, 1976.

13. M. Scharlemann and L. Siebenmann, The Hauptvermutung for smooth singular homeomorphisms, Manifolds (Tokyo, 1973), Univ. of Tokyo Press, 1975, pp. 85-91.

14. M. Shiota, Classification of Nash manifolds, Ann. Inst. Fourier (Grenoble) 33 (1983) 209-232.

15. , Pieceurse linearization of real analytic functions, Publ. RIMS, Kyoto Univ. (to appear).

16. L. C. Siebenmann, Topological manifolds, Actes Congrès Internat. Math. (Nice, 1970), vol. 2, Gauthier-Villars, Paris, 1971, pp. 133-163.

17. J. L. Verdier, Stratifications de Whitney et theéorème de Bertini-Sard, Invent. Math. 36 (1976), 295-312.

RESEARCh InStitute for Mathematical SCIENCES, Kyoto UNiversity, Kyoto 606. JAPAN

Current address: Department of Mathematics, College of General Education, Nagoya University, Nagoya 464, Japan 